\title{
Effects of Flow Structure Dynamics on Thermoacoustic Instabilities in Swirl-Stabilized Combustion
}

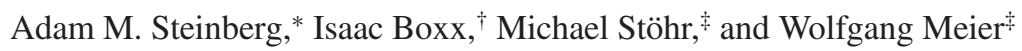 \\ DLR, German Aerospace Center, 70569 Stuttgart, Germany \\ and \\ Campbell D. Carter $\stackrel{\S}{-}$ \\ U.S. Air Force Research Laboratory, Wright-Patterson Air Force Base, Ohio 45433 \\ DOI: $\underline{10.2514 / 1 . J 051466}$
}

\begin{abstract}
The thermoacoustic coupling caused by dynamic flow/flame interactions was investigated in a gas-turbine model combustor using high-repetition-rate measurements of the three-component velocity field, $\mathrm{OH}$ laser-induced fluorescence, and $\mathrm{OH}^{*}$ chemiluminescence. Three fuel-lean, swirl-stabilized flames were investigated, each of which underwent self-excited thermoacoustic pulsations. The most energetic flow structure at each condition was a helical vortex core that circumscribed the combustor at a frequency that was independent of the acoustics. Resolving the measurement sequence with respect to both the phase in the thermoacoustic cycle and the azimuthal position of the helix allowed quantification of the oscillatory flow and flame dynamics. Periodic vortex/flame interactions caused by deformation of the helices generated local heat-release oscillations having spatially complex phase distributions relative to the acoustics. The local thermoacoustic coupling, determined by statistically solving the Rayleigh integral, showed intertwined regions of positive and negative coupling due to these vortices. In the quietest flame, the helical vortex created a large region of negative coupling that helped damp the oscillations. In the louder flames, the shapes of the oscillating vortices and flames were such that large regions of positive coupling were generated, driving the instability. From these observations, flame/vortex configurations that promote stability are identified.
\end{abstract}

\section{Nomenclature}

$a$
.$a p$
$D$
$f$
$M$
$P_{\mathrm{th}}$
$p$
$\dot{q}$
$S$
$\cdot t$
$\dot{v}$
$\beta$
$\Theta$
$\vartheta$
$\theta$
$\lambda$
$\Sigma$
$\phi$
$\Psi$
$\psi$
$\omega$
$\leftrightarrow$

$=$
$=$
$=$
$=$
$=$
$=$
$=$
$=$
$=$
$=$
$=$
$=$
$=$
$=$
$=$

proper orthogonal decomposition temporal coefficient

= doubly-phase-resolved mean oscillation

$=$ dissipation rate of acoustic energy

$=$ frequency

$=$ proper orthogonal decomposition spatial eigenmode

$=$ thermal power

$=$ pressure

$=$ heat-release rate

$=$ swirl number

$=$ excess stochastic turbulent fluctuation

$=$ volume flow rate

$=$ thermoacoustic phase shift

$=$ total thermoacoustic energy transfer

$=$ local thermoacoustic energy transfer

$=$ azimuthal angle through helical vortex cores

$=$ proper orthogonal decomposition eigenvalue

$=$ flame surface density

$=$ phase angle

$=$ total doubly-phase-resolved thermoacoustic coupling

$=$ local doubly-phase-resolved thermoacoustic coupling

$=$ vorticity

$=$ long time average

Presented at the 46th Joint Propulsion Conference \& Exhibit, Nashville, TN, 28-30 2010; received 30 June 2011; revision received 10 August 2011; accepted for publication 10 August 2011. Copyright (C) 2011 by DLR, German Aerospace Center. Published by the American Institute of Aeronautics and Astronautics, Inc., with permission. Copies of this paper may be made for personal or internal use, on condition that the copier pay the $\$ 10.00$ per-copy fee to the Copyright Clearance Center, Inc., 222 Rosewood Drive, Danvers, MA 01923; include the code 0001-1452/12 and $\$ 10.00$ in correspondence with the CCC.

${ }^{*}$ Research Associate, Institute for Combustion Technology; currently Assistant Professor, Institute for Aerospace Studies, University of Toronto. Member AIAA.

${ }^{\dagger}$ Research Associate, Institute for Combustion Technology. Member AIAA.

*Research Associate, Institute for Combustion Technology.

§Principal Aerospace Engineer, Advanced Propulsion Division. Associate Fellow AIAA.

$$
\stackrel{\leftrightarrow}{a p}=\begin{aligned}
& \text { sum of long average and doubly-phase-resolved } \\
& \text { oscillation }
\end{aligned}
$$

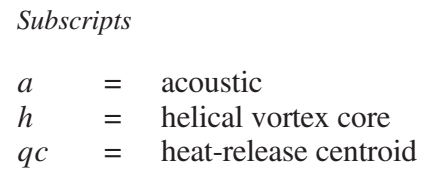

\section{Introduction}

C OMBUSTION-DRIVEN pressure and heat-release oscillations, or thermoacoustic instabilities, are a major problem in low-emission gas-turbine engines [1-4] . These instabilities are naturally excited by feedback loops that couple an acoustic mode of the engine with a process causing variations in the rate of heat release from combustion. The resultant large-amplitude pressure and heatrelease oscillations lead to corresponding oscillations in the mechanical and thermal loads on various engine components, causing premature (and possibly catastrophic) component failure. Other problems, such as flame blowoff and flashback, reduced combustion efficiency, and increased pollutant emissions, also can be caused by thermoacoustic instabilities. Although different control strategies have been implemented with varying degrees of success [4-8], these are generally applied as retrofits when unstable conditions are encountered late in the engine testing process, and they are not robust to configuration changes, fuel changes, wide operation ranges, or unexpected transients. It is therefore highly desirable to better understand the mechanisms coupling the acoustics and heat release in order to better predict, avoid, and control thermoacoustic instabilities. In this work, these mechanisms are analyzed in a model gasturbine combustor based on data from high-repetition-rate laser measurements.

Thermoacoustic coupling occurs when a natural acoustic oscillation in the engine causes, through some mechanism, an oscillation in the heat-release rate. Thermoacoustically unstable states exist when such couplings cause the total amount of energy transferred from unsteady combustion to the acoustic field $\Theta$ over a given time to exceed the amount of acoustic energy that is dissipated within the combustor or transmitted through its boundaries $D$. The 
local acoustic energy transfer from the thermoacoustic coupling $[\vartheta(\mathbf{x})]$ is determined by the local integral of the pressure $p$ and volumetric heat-release $\dot{q}$ fluctuation, with the net energy transfer given by the integral over the combustor volume:

$$
\Theta=\int_{V} \vartheta(\mathbf{x}) \mathrm{d} V=\int_{V} \int_{T} p^{\prime}(\mathbf{x}, t) \dot{q}^{\prime}(\mathbf{x}, t) \mathrm{d} t \mathrm{~d} \mathbf{x}
$$

This is referred to as the Rayleigh integral and shows that energy is locally transferred to the acoustic field when the phase shift between the heat release and the pressure oscillations is less than $90^{\circ}$. Otherwise, energy is transferred away from the acoustic field. The system is unstable and the oscillations grow when $\Theta>D$. Earlydesign prediction of thermoacoustically unstable conditions therefore requires prediction of the heat-release fluctuation distribution and its local phase shift relative to the combustor acoustics.

This is not yet possible in practical systems due to the complex thermofluidic processes required to stabilize low-emission, fuel-lean flames in gas-turbine combustors. A common flow configuration for flame stabilization in low-emission combustors involves swirling premixed or partially premixed reactants tangentially around the nozzle axis [4,9-12]. Swirl-induced vortex breakdown in these combustors leads to the formation of a large central recirculation zone (CRZ) downstream of the nozzle exit that transports hot products back to the flame root, stabilizing the combustion $[13,14]$. The swirling flow also may lead to the formation of unsteady, largescale, helical vortex cores (HVCs) $[10,13,15-24]$. These threedimensional dynamic flow structures circumscribe the nozzle and affect the mixing and combustion processes. Periodic heat-release oscillations therefore do not occur simultaneously everywhere in the combustor or in radially symmetric patterns, but instead have complex amplitude and phase distributions that are determined by the local dynamic interaction of flow structures with the flame and acoustics. Furthermore, the behavior, shape, and even existence of such structures can depend on the combustor and acoustics. In recent measurements, Lacarelle et al. [23] found that helical modes that were present in an unforced swirl flame did not occur when the flow was forced at the natural frequency of the combustor. However, these modes reappeared when the system was forced at higher frequencies. Computational studies have shown that helical structures that are present in nonreacting flows can be damped by combustion [13] or low-frequency oscillations [24].

In this work, swirl-flow/flame interactions, their effect on the heat release, and the resultant thermoacoustic coupling are investigated in a gas-turbine model combustor (GTMC). The burner is a modified version of a practical gas-turbine combustor that was operated with swirl-stabilized, partially premixed, methane-air flames at atmospheric pressure. Such GTMC conditions bridge the gap between fundamental combustion experiments and large-scale experiments at industrial conditions, allowing flames that exhibit many features of practical systems to be studied in well-controlled, rigorously defined, and highly repeatable laboratory conditions. Furthermore, GTMC flames allow for the application of many advanced laser diagnostics techniques that are impractical at industrial operating conditions, thus providing significant physical insight and detailed model validation data.

Several experimental investigations have previously been conducted on this burner over a range of operating conditions and using various laser-based diagnostics [14,25-32]. Under some conditions, flames in this combustor undergo large-amplitude selfexcited thermoacoustic oscillations, whereas under other conditions, the flames operate stably. A CRZ and HVC have been observed at all conditions, regardless of the thermoacoustic behavior. This burner therefore provides an optimal configuration with which to study the influence of these flow features on thermoacoustic instabilities. Here, data from high-repetition-rate laser diagnostics are used to study thermoacoustic coupling in three flames with various amplitude thermoacoustic pulsations.

The flame structure in this burner was elucidated by Weigand et al. [14] and Meier et al. [26] using $\mathrm{OH} / \mathrm{CH}$ planar laser-induced fluorescence (PLIF), laser Doppler velocimetry, and Raman scattering. It was found that the flame did not stabilize directly on the fuel nozzle, but was lifted by several millimeters, allowing the fuel and air to partially premix before combustion began at the flame root. The combustion behavior in the near field of the nozzle was influenced both by mixing and finite rate chemistry. Reactions occurred in thin layers and strong turbulence-chemistry interactions were observed to cause local corrugation and extinction of these layers.

Such interactions were further elucidated by Sadanandan et al. [27] and Stöhr et al. [28] using stereoscopic particle image velocimetry (PIV) and OH-PLIF. It was shown that the dominant flow structure was a helical vortex that circumscribed the burner nozzle at a frequency that was independent of the acoustics. Considerable mutual interaction between this HVC and flame was observed. Note that in previous work, this vortical structure was referred to as a precessing vortex core. However, precession of the vortex core about the nozzle axis (i.e., change in the orientation of the rotation axis) describes the motion of only a segment of the structure at the nozzle exit. The term HVC is used in this work as we will be interested in the dynamics of the entire flow structure, the majority of which rotates about the central axis.

Recently, Boxx et al. [29] and Stöhr et al. [30] studied this combustor using high-repetition-rate laser diagnostics. Stereoscopic PIV, OH-PLIF, and $\mathrm{OH}^{*}$ chemiluminescence diagnostics all were applied at a sustained repetition rate of $5 \mathrm{kHz}$. This allowed qualitative observation of several important flow/flame interactions, including flame roll-up, local extinction, and possible autoignition events. However, the high through-plane motion caused by the swirling flow leads to difficulty in interpreting individual time sequences; the measurements at a given time are not the continuation of the same fluid and flame that were measured at the previous time. It is therefore not possible to directly determine if the observed events are due to transient interactions or through-plane convection of quasi-stable configurations. However, Steinberg et al. [31] showed that signals from the temporally resolved planar measurements could be used to identify the repeatable flow and flame dynamics in such swirl flames. In this work, the analysis and diagnostic techniques that were previously developed are used to rigorously study the influence of complex, three-dimensional, flow/flame interactions on thermoacoustic coupling.

\section{Experiment and Diagnostics}

The gas-turbine model combustor used for this work has been the subject of numerous previous studies in which the geometry is described in detail $[14,25-28]$. Furthermore, the analysis presented in this work is based on the experiments of Boxx et al. [29]. Hence, only a brief description of the combustor and diagnostics is provided here.

\section{A. Gas-Turbine Model Combustor}

The combustor is shown schematically in Fig. 1. Coswirling dry air at room temperature was supplied to the flame through a central nozzle (diameter $15 \mathrm{~mm}$, eight swirl channels) and an annular nozzle (i.d. $17 \mathrm{~mm}$, o.d. $25 \mathrm{~mm}$ contoured to an o.d. of $40 \mathrm{~mm}, 12$ swirl channels), both of which were fed from a common plenum. Nonswirling $\mathrm{CH}_{4}$ was introduced through a ring composed of 72 square $0.5 \times 0.5 \mathrm{~mm}$ channels between the air nozzles. The exit plane of the fuel and central air nozzles was $4.5 \mathrm{~mm}$ below that of the outer nozzle, and the latter was taken as the reference height $(y=0)$.

The burner was enclosed in an optically accessible combustion chamber composed of fused-silica plates held in the corners by Inconel ${ }^{\circledR}$ alloy posts. This allowed virtually unobstructed optical access to the flame. The chamber had a square cross section of $85 \times 85 \mathrm{~mm}$ and a height of $114 \mathrm{~mm}$. The exhaust was formed from a steel plate with a conical contraction, leading to an exhaust tube with $40 \mathrm{~mm}$ diameter.

Flames in this burner often exhibit self-excited thermoacoustic pulsations, the magnitude of which depends on the specific flow conditions; under some conditions, the pressure and heat-release fluctuations couple to produce large-amplitude pulsations, and under other conditions, the burner operates relatively stably $[\underline{14}, 26]$. Three 


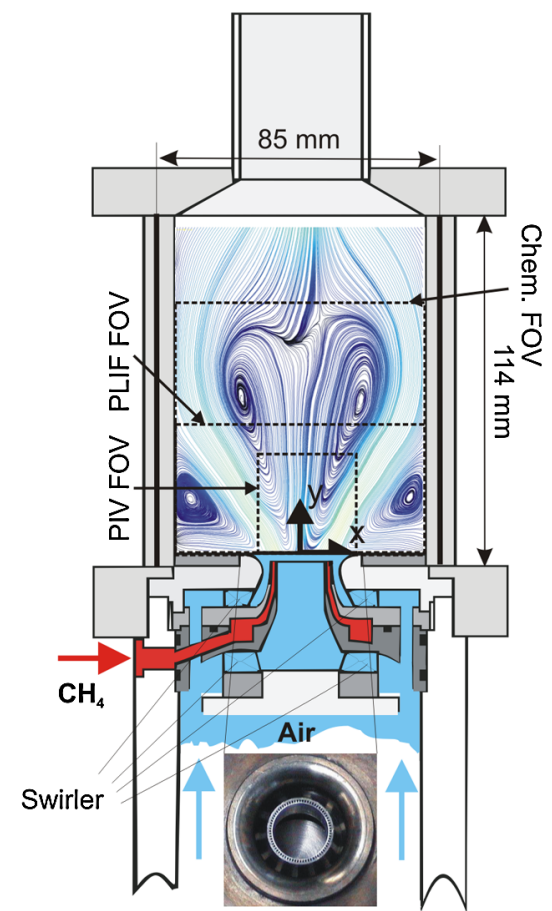

Fig. 1 Gas-turbine model combustor with the fields of view for the various diagnostics. The streamlines indicate the mean velocity field for flame 3 measured in Sadanandan et al. [27].

different flames (summarized in Table 1) are studied here, each of which underwent thermoacoustic pulsations of different amplitudes. Each flame had a single pulsation amplitude. Flame 1 had a thermal power of $P_{\text {th }}=7.6 \mathrm{~kW}$, an equivalence ratio of $\phi=0.55$, and was close to the lean blowoff limit. This flame would periodically (1-2 times per second) lift off of the burner nozzle for approximately $0.1 \mathrm{~s}$ and then reanchor. The liftoff and reanchoring has been investigated elsewhere and is not the focus of the present work [30]. Instead, only temporal segments during which the flame was stably attached to the nozzle will be used. Such temporal segments were typically between 0.5 and $1 \mathrm{~s}$ in duration and therefore provided several thousand frames of continuous data from the high-repetition-rate diagnostics. Furthermore, all processes in the combustor during these time segments were steadily periodic at distinct frequencies. Flame 1 was the quietest flame studied, with a root-mean-square (rms) pressure fluctuation magnitude of $p^{\prime}=85 \mathrm{~Pa}(132.6 \mathrm{~dB})$ at a frequency of $f_{a}=302 \mathrm{~Hz}$. Flame 2 was operated at $P_{\mathrm{th}}=10 \mathrm{~kW}$ and $\phi=0.65$. This flame exhibited slightly stronger pressure oscillations of $p^{\prime}=$ $130 \mathrm{~Pa}(136.3 \mathrm{~dB})$ at $305 \mathrm{~Hz}$. Flame 3 also was operated at $P_{\mathrm{th}}=10 \mathrm{~kW}$, but with an equivalence ratio of $\phi=0.75$, and underwent stronger thermoacoustic pulsations of $p^{\prime}=220 \mathrm{~Pa}$ $(140.8 \mathrm{~dB})$ at $308 \mathrm{~Hz}$. The swirl number for all flames was estimated in earlier work from the ratio of the tangential and axial momentum fluxes as $S=0.55$. Detailed measurements of the boundary conditions and thermochemical state have been performed for flames 1 and 3 using laser Doppler velocimetry and Raman scattering and are available from the authors for simulation development and validation $[14,26]$.

All of the flow rates $\dot{m}$ listed in Table 1 were controlled using electromechanical mass flow controllers (Brooks) and monitored using calibration standard Coriolis mass flow meters (Siemens Sitrans F C) with an uncertainty of $1.5 \%$. For all cases, the burner was

Table 1 Flow and flame conditions

\begin{tabular}{ccccccc}
\hline \hline Flame & $P_{\text {th }}, \mathrm{kW}$ & $\phi$ & $\dot{m}_{\text {air }}, \mathrm{g} / \mathrm{s}$ & $\dot{m}_{\mathrm{CH} 4}, \mathrm{~g} / \mathrm{s}$ & $p^{\prime}, \mathrm{Pa}$ & $f_{a}, \mathrm{~Hz}$ \\
\hline 1 & 7.6 & 0.55 & 4.7 & 0.15 & 85 & 302 \\
2 & 10 & 0.65 & 5.4 & 0.20 & 130 & 305 \\
3 & 10 & 0.75 & 4.7 & 0.20 & 220 & 308 \\
\hline \hline
\end{tabular}

allowed to thermally stabilize at full power for at least $20 \mathrm{~min}$ before data acquisition. During a data-acquisition run, approximately $4 \%$ of the air mass flow was diverted through a fluidized bed particle seeder containing $1 \mu \mathrm{m} \mathrm{TiO}_{2}$ particles to enable the velocity measurements. The airflow was seeded only during the short data-acquisition periods to reduce the accumulation of particles on the windows. Because the flow was seeded for only a few seconds at a time, window degradation was minimized and several data-acquisition runs could be accomplished before window contamination significantly affected the measurements.

The system was equipped with multiple ports for microphone probes in the corner posts of the combustion chamber and in the plenum wall. Figure 2 shows the mean pressure oscillation, measured using calibrated microphone probes (Brüel \& Kjaer, Type 4939), at several locations for flame 3 . As can be seen, the shape, amplitude, and phase of the pressure oscillation was essentially identical everywhere in the combustion chamber. The plenum pressure signal lagged the combustion-chamber signals by approximately $60^{\circ}$. The pressure signals in flames 1 and 2 followed similar trends, which is consistent with a multichamber Helmholtz resonator mode. Acoustic simulations using Comsol have confirmed this resonation to be the dominant mode, with the ratio of the plenum to chamber pressure amplitudes peaking when the oscillations occur at the natural frequency of the total system. A detailed analysis of the acoustic eigenmodes relative to the flow-structure dynamics will be the subject of future work.

Because of the similarity of the signals, only two probes were used during the laser and optical measurements; one was mounted in a corner post of the combustion chamber and one was in the outer wall of the plenum. Both probes were sampled at a rate of $20 \mathrm{kHz}$ using a multichannel data-acquisition system, which simultaneously recorded the camera intensifier trigger for the PLIF system described below. This allowed synchronization of the acoustic and laser-based measurements. In all cases, the pressure signals recorded by the microphone probes in the combustion chamber and air plenum had essentially identical frequency spectra. The signal from the plenum microphone exhibited less noise than that from combustion chamber, but was shifted in phase by between $60-80^{\circ}$ (determined from the dominant frequency in the Fourier transformation), depending on the flame. Because of the reduced noise, this plenum signal was shifted in phase to match that in the combustion chamber and used to identify the phase angle in the acoustic cycle at which each laser-based measurement was acquired.

\section{B. Stereoscopic Particle Image Velocimetry}

Three-component planar velocity fields were measured at a rate of $5 \mathrm{kHz}$ using stereoscopic PIV. The system, shown in Fig. 3, consisted of a high-repetition-rate, dual-cavity, diode-pumped, solid-state $\mathrm{Nd}$ : YAG laser (Edgewave, IS-6IIDE) and a pair of high-speed complementary metal-oxide semiconductor (CMOS) cameras (LaVision

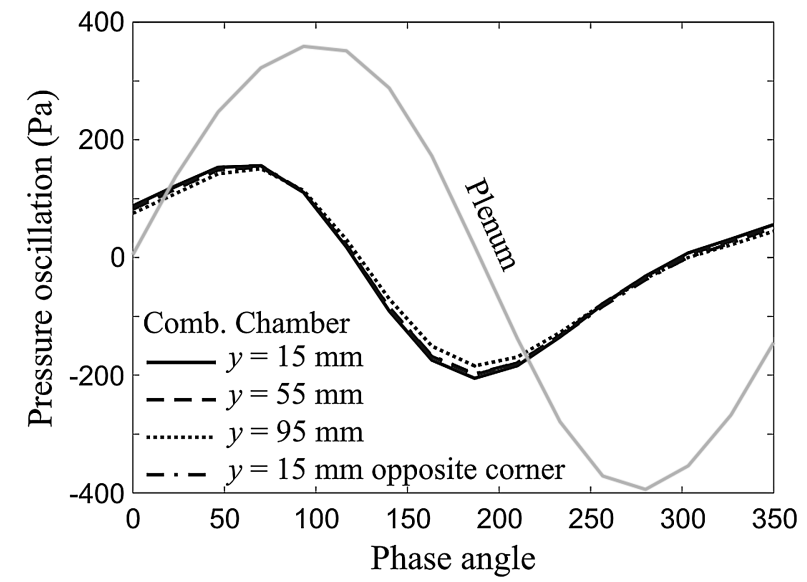

Fig. 2 Mean pressure oscillation in combustion chamber and plenum for flame 3 . 


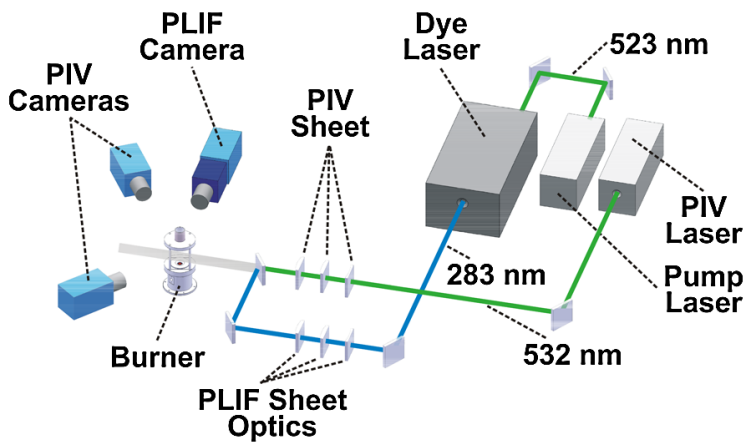

Fig. 3 Layout of simultaneous $5 \mathrm{kHz}$ stereoscopic PIV and OH-PLIF system.

HSS5). Laser pulse pairs (532 nm, $2.6 \mathrm{~mJ} /$ pulse, $14 \mathrm{~ns}$ pulse duration, $20 \mu$ s between pulses) repeating at $5 \mathrm{kHz}$ were expanded into a collimated sheet using a two-component cylindrical-lens telescope. The sheet was then focused to a width of $0.7 \mathrm{~mm}$ at the burner axis using a third cylindrical lens. Particle-scattered light from the $\mathrm{TiO}_{2}$ seed was collected into the cameras using 100-mm-focallength commercial camera lenses set to $f 5.6$ (Tokina).

The pair of CMOS cameras were mounted equidistantly from opposite sides of the laser sheet in a forward-scatter configuration. Image defocusing due to the angular configuration was corrected by rotating the camera body with respect to the lens as stipulated by the Scheimpflug criterion. Perspective distortion brought about by this configuration was corrected by imaging a three-dimensional dot target (LaVision type 7) that was placed in the measurement plane. The distorted dot target was transformed to a normal coordinate system using the LaVision DaVis 7.2 software package. The same target images were used to align the fields of view from the PIV cameras with that from the PLIF camera described below.

Vector fields were computed from the particle image spatial crosscorrelation using the LaVision DaVis 7.2 software package. An adaptive multipass vector evaluation technique was used, with interrogation boxes ranging from 64 to 16 pixels. In dual-frame mode at $5 \mathrm{kHz}$, the PIV cameras had an active sensor size of $512 \times$ 512 pixels. The field of view imaged by the PIV system was $32 \times$ $30 \mathrm{~mm}$ (shown in Fig. 1), resulting in a spatial resolution and vector spacing of approximately 0.94 and $0.47 \mathrm{~mm}$, respectively. Each camera had 2.6 GB of memory, allowing 4096 image pairs to be obtained over a measurement duration of approximately $0.8 \mathrm{~s}$.

\section{Planar Laser-Induced Fluorescence and Chemiluminescence}

Laser-induced fluorescence was used to measure the planar distribution of the $\mathrm{OH}$ combustion radical and was conducted simultaneously with the PIV measurements. The OH-PLIF system consisted of a high-repetition-rate, frequency-doubled Nd:YLF laser (Edgewave IS-8IIE) pumping a dye laser (Sirah Cobra-Stretch modified for kilohertz pumping). At $5 \mathrm{kHz}$, the pump laser delivered $3.8 \mathrm{~mJ} /$ pulse ( $19 \mathrm{~W}$ average output) at $523 \mathrm{~nm}$ with an $8.5 \mathrm{~ns}$ pulse duration. The output of the dye laser was frequency-doubled and tuned to excite the $Q_{1}(7)$ line of the A-X $\left(v^{\prime}=1, v^{\prime \prime}=0\right)$ transition of $\mathrm{OH}$ at $283.2 \mathrm{~nm}$. Tuning of the laser wavelength was checked daily using a calibration burner. After frequency doubling, the average output power of the dye laser at $283.2 \mathrm{~nm}$ was $0.5 \mathrm{~W}$ or $0.1 \mathrm{~mJ} /$ pulse. The laser beam was formed into a sheet with a height of approximately $40 \mathrm{~mm}$ and a waist of approximately $0.4 \mathrm{~mm}$ using three cylindrical lenses. The pixel resolution of the camera was considerably smaller than the sheet thickness, which set the limiting resolution. This sheet was overlapped with the PIV laser sheet using a dichroic mirror and sent into the test section along the same beam path.

Fluorescence of the $\mathrm{OH}$ radical in the range of $310 \mathrm{~nm}$ was acquired with a CMOS camera (LaVision HSS6) equipped with an external, two-stage, lens-coupled intensifier (LaVision HS-IRO) and a $45 \mathrm{~mm}$ f1.8 UV lens (Cerco). The intensifier gate time was set to
$500 \mathrm{~ns}$ and the PLIF laser pulse occurred between the first and second PIV laser pulses on each cycle. The field of view imaged by the PLIF system covered the entire width of the combustion chamber $(85 \mathrm{~mm})$ and extended from the nozzle exit to a height of $40 \mathrm{~mm}$.

Background luminosity and elastic scattering were reduced by using a 500 ns intensifier gate, a high-transmission (greater than $80 \%$ at $310 \mathrm{~nm}$ ) bandpass interference filter (Laser Components $\mathrm{GmbH}$ ), and a color glass filter (1-mm-thick WG295 Schott glass). The $\mathrm{OH}$ images were corrected for the mean laser sheet intensity profile, which was determined based on 1000 images of the fluorescence from a uniform acetone vapor that was doped into the test area. A correction also was made to remove the mean flame luminosity. The PLIF images were filtered with a $0.4 \mathrm{~mm}$ Gaussian filter (same width as the sheet thickness) before further analysis to reduce highfrequency pixel noise.

Since the chemical reactions in these flames occur in thin layers [14], the local heat-release rate is the product of the flame surface area and the reaction rate per unit area. However, $\mathrm{OH}$ radicals exist not only in regions of heat release (i.e., the flame surfaces), but also in hot gases above a temperature of approximately $1500 \mathrm{~K}$ [27]. The PLIF signal from $\mathrm{OH}$ radicals therefore exists as broadly distributed regions. The topography of the flame surface can be mapped from these regions due to the fact that the $\mathrm{OH}$ signal increases rapidly in the heat-release zone to superequilibrium concentrations before relaxing back to equilibrium at a relatively slow rate in the postflame gases. Hence, the flame surface is associated with regions of high $\mathrm{OH}$ gradient. Steinberg et al. [31] developed a robust algorithm to determine the flame topography and reduce it to mathematical contours by simultaneously considering the $\mathrm{OH}$ signal, gradient, and profile curvature. This algorithm is used here to compute the topography of the reaction layers, each of which was treated as an individual parametrically defined contour, $f(\varphi)=x_{f}(\varphi) \hat{e}_{x}+$ $y_{f}(\varphi) \hat{e}_{y}$, where $\hat{e}_{x}$ and $\hat{e}_{y}$ are the respective unit vectors in the radial and axial directions. The algorithm is able to detect and disclude locally extinguished regions and nonburning interfaces between hot gas mixtures and reactants. It is noted that, although the flame area may be determined from the computed flame topographies, the reaction rate per unit area may vary along the flame due to local stratification (i.e., nonhomogeneous equivalence ratio), strain rate, and preheating due to product-gas recirculation. Nevertheless, the derived topographies are sufficient to study many mechanisms of thermoacoustic instability.

Additionally, the line-of-sight integrated spontaneous emission of electronically excited $\mathrm{OH}\left(\mathrm{OH}^{*}\right)$ was imaged separately from the PLIF and PIV measurements, but under identical run conditions. The camera/lens/filter arrangement for the chemiluminescence camera was identical to that of the OH-PLIF camera; however, the intensifier gate time was extended to $25 \mu \mathrm{s}$ in order to capture sufficient signal. The field of view was not restricted by a laser sheet height, and chemiluminescence was collected over an $85 \times 85 \mathrm{~mm}$ region at a rate of $5 \mathrm{kHz}$ for a $0.8 \mathrm{~s}$ duration. Naturally excited $\mathrm{OH}$ exists only within the thin reaction layers, and the chemiluminescence signal can be taken as a qualitative indicator of the integrated heat-release rate in the combustor [5]. Furthermore, although there are definite uncertainties in using chemiluminescence as a quantitative or spatially resolved measurement, particularly in turbulent stratified flows, it is a sufficiently accurate marker of the heat-release location for the purposes of the following analysis [33-36].

\section{Temporally Resolved Flow and Flame Dynamics}

Steinberg et al. [31] present several short time sequences of velocity and $\mathrm{OH}$ fields that demonstrate important flow/flame interactions in flame 3 . These include an oscillating reactant flux due to coupling of the combustion-chamber pressure with the plenum, large-scale wrap-up of the flame by a coherent vortical structure, asymmetric reactant inflow and combustion driven by this coherent structure, and small-scale corrugation of the flame by stochastic turbulence.

To quantify the reactant flux oscillations, the volumetric flow rate through the $y=0$ plane $(\dot{v})$ was computed by instantaneously 
Table 2 Properties of flowfield fluctuations

\begin{tabular}{ccccc}
\hline \hline Flame & $f_{a}, \mathrm{~Hz}$ & $\dot{v} / \stackrel{\leftrightarrow}{v}$ & $f_{h}, \mathrm{~Hz}$ & $f_{q c}, \mathrm{~Hz}$ \\
\hline 1 & 302 & 0.25 & 507 & 204 \\
2 & 305 & 0.45 & 555 & 250 \\
3 & 308 & 0.55 & 515 & 207 \\
\hline \hline
\end{tabular}

integrating the positive axial velocity across the nozzle exit. Magnitudes of the rms volume flux oscillations normalized by the long time averages taken over hundreds of thermoacoustic cycles, denoted by $(\stackrel{\leftrightarrow}{\circ}$ ), are listed in Table 2 . As can be seen, the pressure oscillations caused large flow-rate oscillations, reaching values of over $50 \%$ of the mean in flame 3 .

Furthermore, it was previously demonstrated that the observed coherent structure in flame 3 was a large-scale HVC that circumscribed the burner at a frequency that was different from and greater than that of the thermoacoustic pulsations $\left(f_{h}>f_{a}\right)[29,31]$. However, the HVC underwent a periodic deformation (i.e., axial extension and contraction) over the thermoacoustic cycle (at $f_{a}$ ). The heat-release centroid $x_{q c}$ circumscribed the burner at the difference between the HVC and thermoacoustic frequencies $\left(f_{q c}=f_{h}-f_{a}\right)$ [31]. The HVC has been observed under all operating conditions studied in this combustor. An HVC also is present in industrial gas turbines and has been shown to greatly affect thermoacoustic instabilities (e.g., Syred [10] and references therein).

The frequency of the flame centroid motion indicates that understanding the interaction of the three-dimensional swirling flow with the flame is essential for understanding the behavior of the heatrelease rate, and consequentially the thermoacoustic coupling. This can be achieved by applying spatiotemporal proper orthogonal decomposition (POD) analysis to the high-repetition-rate velocity measurements. POD analysis is a mathematically rigorous method of extracting the most energetic features of a data set and has been successfully applied to the study of turbulence and combustion $[23,24,29,31,37-41]$. The mathematical basis of POD is well established, and a description of the technique as applied here is given by Steinberg et al. [31]. In summary, POD analysis from a sequence of $n$ temporally resolved velocity-field measurements provides a set of $n$ spatial eigenmodes $M_{j}(x, y)$, temporal coefficients $a_{j}(t)$, and eigenvalues $\lambda_{j}$, such that the eigenmodes form an orthogonal basis for the original data set. The eigenvalues represent the contribution of the modes to the overall kinetic energy of the flow, and the particular property of POD analysis is that it provides optimal convergence of the kinetic energy. That is, the sum of the highest $k$ eigenvalues for the POD basis is larger than for any other orthogonal basis. Assuming that the eigenvalues are sorted in descending order, the first modes represent the dominant flow features. The temporal history of the velocity field from the $k$ most dominant modes is given by

$$
u_{k}\left(x, y, t_{i}\right)=\sum_{j=0}^{k} a_{j}\left(t_{i}\right) M_{j}(x, y)
$$

It is common practice in the application of POD to center the data about the mean, such that the first (most energetic) mode represents a turbulent fluctuation. However, the mean flow pattern for the flames studied here oscillated in intensity due to the coupling of the combustion chamber with the reactant feed system. The POD was therefore performed on the full data set such that $M_{0}(x, y)$ represented the shape of the long-time-average velocity field; $a_{0}(t)$ represented the periodic fluctuation in the reactant flow rate. The temporal history and frequency spectrum of $a_{0}(t)$ were qualitatively the same as those of $\dot{v}(t)$.

The most energetic turbulent modes of the POD for each flame represented the $\mathrm{HVC}$ and its motion around the combustion chamber [31]. Figure 4 shows the frequency spectrum of $a_{1}(t)$ from each flame. Every cycle of these coefficients represents a cycle of the HVC around the combustor from an arbitrary starting point in the measurement plane. Hence, the dominant frequency in the spectrum

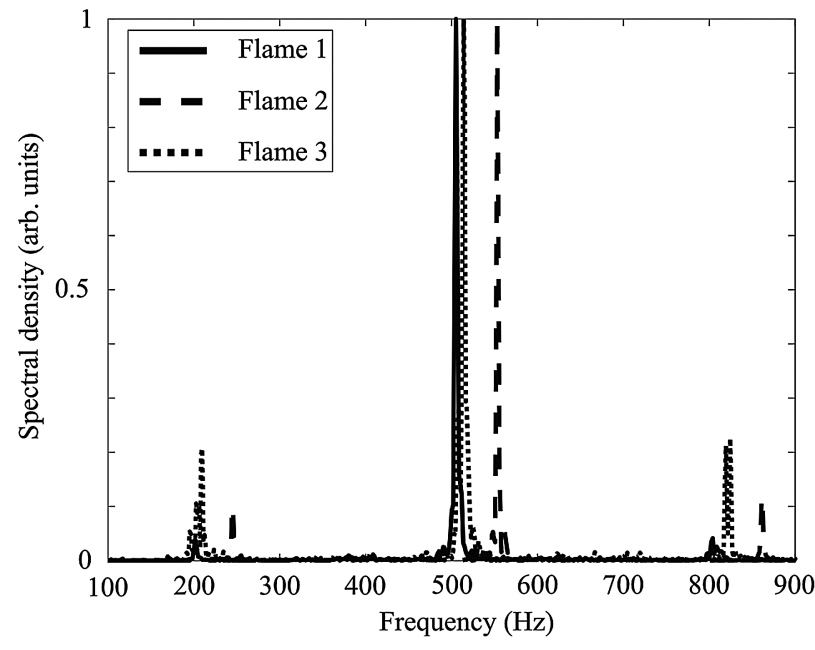

Fig. 4 Frequency spectra of $a_{1}(t)$ indicating the frequency of the HVC motion around the burner in each flame.

indicates the rotation frequency of the HVC. The smaller peaks are at the sum and difference of the thermoacoustic and HVC frequencies, whereas the thermoacoustic frequencies themselves do not appear, because oscillations at $f_{a}$ are represented by different modes of the POD. The HVC frequency, taken as the location of the peaks in Fig. 4, are listed in Table 2. Previous studies of HVCs in nonreacting and unconfined reacting flows have shown that the HVC frequency increases linearly with the total flow rate and also can be affected by the thermochemical properties of the flame [10]. Correspondingly, the HVC frequency in flame 2 was higher than those in flames 1 and 3 due to the higher total flow rate, but the frequencies did not vary in a purely linear manner due to the different global equivalence ratios. Note that the HVC frequency for each flame was very steady, with a full width at half-maximum in the spectral peak of only a few hertz. However, the instantaneous flow rate into the combustion chamber could vary by over $50 \%$ during a thermoacoustic cycle as noted above in the case of flame 3 . This indicates that, although the overall rotation frequency varies linearly with flow rate, it is insensitive to high-frequency flow-rate fluctuations. There was no evidence of widely varying $\mathrm{HVC}$ frequencies in any mode of the POD analysis or from individual measurement sequences.

Figure $\underline{5}$ shows the slice of the HVC that intersected the measurement plane at three different instants during one cycle around the burner for flames 1 and 3. The images were reconstructed from the zeroth-10th modes of the POD, which contained over $70 \%$ of the total turbulent kinetic energy of the flow. Furthermore, over $90 \%$ of the turbulent kinetic energy of modes exhibiting coherent frequencies (in which there were distinct spectral peaks) was contained in this subset. The remaining modes generally represented stochastic turbulence in which the energy was distributed over a range of frequencies. Hence, these images represent the dynamics of the periodic flow structures with the majority of the stochastic turbulence removed.

The rotation of these HVCs around the burner had approximately the same period of around $2 \mathrm{~ms}\left(f_{h} \approx 500 \mathrm{~Hz}\right)$. In both cases, the HVC began with its lowest spiral on the left side of the measurement plane, moved approximately $180^{\circ}$ around the burner in $1 \mathrm{~ms}$, and then returned to its original azimuthal position in $2 \mathrm{~ms}$. In the case of flame 1, the axial distance between the helix branches that intersect the measurement plane was constant throughout the time sequence. In flame 3, however, the helical vortex underwent significant contraction in the axial direction; when the HVC returned to its original azimuthal position, the axial distance between the branches was reduced. It will be demonstrated in Sec. V.B that these axial dynamics are periodic over the thermoacoustic cycle, not the HVC motion around the burner. It also will be demonstrated that the magnitude of the axial deformation is dependent on the magnitude of the thermoacoustic oscillation. The HVC in flame 1 exhibited lowamplitude axial dynamics, the HVC in flame 2 exhibited moderate 


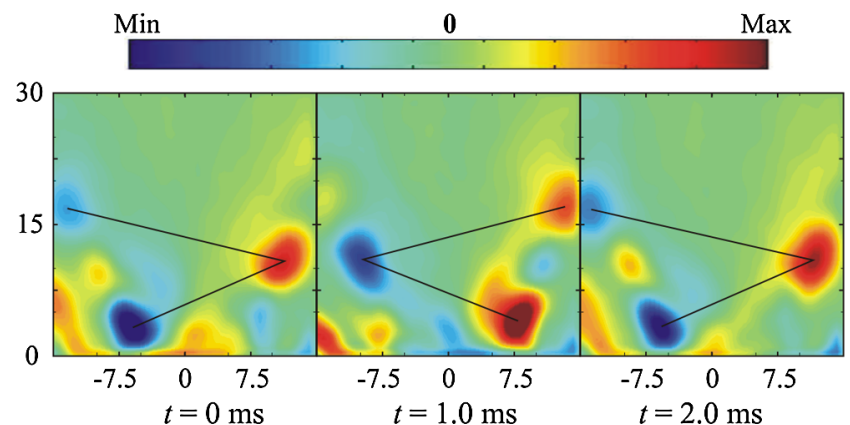

a) Flame 1

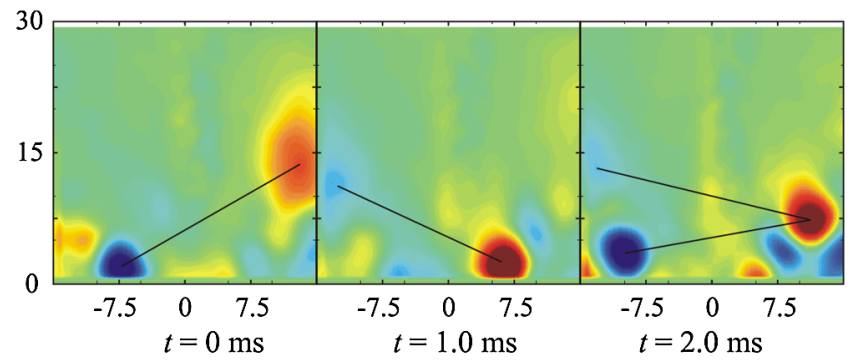

b) Flame 3

Fig. 5 Dynamics of the helical vortex core reconstructed from the zeroth-10th modes of the POD in flames 1 and $3\left(u_{10}(t)\right)$, both of which had frequencies of approximately $500 \mathrm{~Hz}$. The HVCs circumscribed the nozzle in $2 \mathrm{~ms}$ and the HVC for flame 3 exhibited axial dynamics that were periodic over the thermoacoustic cycle. The flow is visualized in terms of the resolved vorticity $\omega_{z}$ between $-20,000 \mathrm{~s}^{-1}$ and $20,000 \mathrm{~s}^{-1}$. Axes units are in millimeters.

amplitude dynamics, and the HVC in flame 3 exhibited the largestamplitude dynamics.

As previously shown by Steinberg et al. [31] and mentioned above, the $\mathrm{OH}^{*}$ chemiluminescence measurements in flame 3 showed that the heat-release centroid circumscribed the burner at $f_{q c}=207 \mathrm{~Hz}$, which can now be identified as the difference between the HVC frequency $\left(f_{h}=515 \mathrm{~Hz}\right)$ and the thermoacoustic frequency $\left(f_{a}=308 \mathrm{~Hz}\right)$. The power spectrum of the radial heatrelease centroid location for each flame is shown in Fig. 6 and the frequency of the spectral peaks are listed in Table 2 . As in flame 3, the heat-release centroid in flames 1 and 2 circumscribed the burner at the difference between their respective $\mathrm{HVC}$ and thermoacoustic frequencies. Furthermore, the amplitude of the radial centroid motion increased from flame 1 to flame 3. It will be shown in Sec. V.A that this motion is related to the axial dynamics of the HVC $\overline{\text { and }}$ therefore increases with thermoacoustic amplitude.

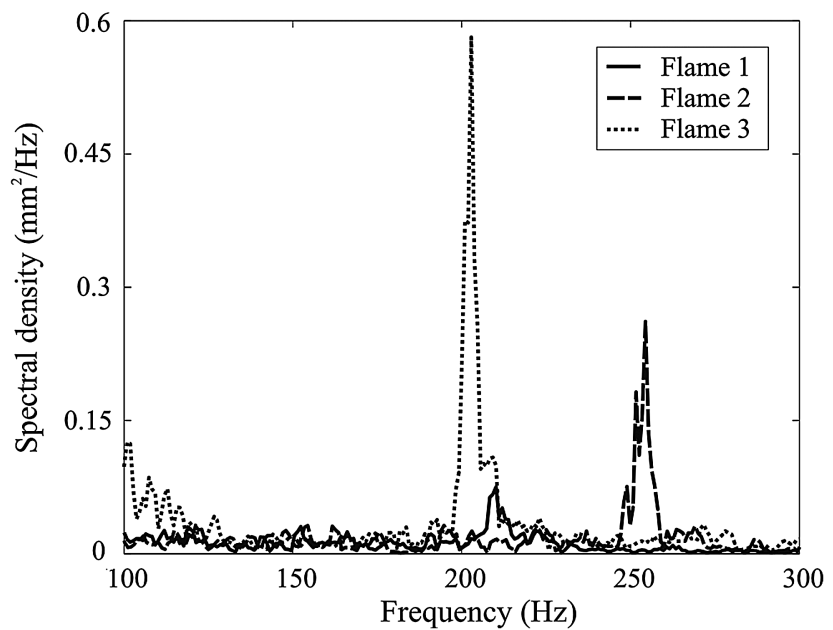

Fig. 6 Power spectra of the radial location of the intensity-weighted heat-release centroid in all flames $\left(x_{q c}\right)$.

\section{Doubly-Phase-Resolved Analysis}

Since the interaction of the HVC and the flame over an acoustic cycle has a large effect on the heat-release rate, correctly interpreting the flow and combustion from planar measurements requires simultaneous consideration of both the pressure fluctuation and the azimuthal position of the HVC with respect to the measurement plane. This can be achieved by resolving the temporal measurement sequence with respect to the phase of both phenomena [31]. The phase in the acoustic oscillation $\left(\phi_{a}\right)$ was determined from the plenum microphone measurements, and phase of the HVC motion $\left(\phi_{h}\right)$ was measured from the temporal coefficient of the first POD mode, which was sinusoidally periodic over the HVC motion around the combustion chamber [31]. For each process, the time at which the signal crossed zero from positive to negative was taken as the beginning of a cycle. Any measured variable $\zeta$ can then be decomposed into three parts, a long time average $\stackrel{\leftrightarrow}{\zeta}$, a doubly-phaseresolved mean oscillatory component $\zeta^{a p}\left(\phi_{a}, \phi_{h}\right)$, and a turbulent fluctuation $\zeta^{t}(t)$, such that

$$
\zeta(t)=\overleftrightarrow{\zeta}+\zeta^{a p}\left(\phi_{a}, \phi_{h}\right)+\zeta^{t}(t)
$$

Note that the HVC phase angle is a measure of the position of the HVC from an arbitrary starting point relative to the measurement plane. The choice of this starting point does not affect the results as long as a consistent definition is used.

For the purposes of statistical analysis, each cycle of each process was divided into eight discrete phase angles, shown in Fig. 7, and the actual phase angles of the instantaneous measurements correlated with the closest combination of discrete phase angles. This resulted in 64 phase-angle combination pairs describing the repeatable combustor dynamics. Throughout the remainder of the text, all phase angles will be given in terms of the numbering scheme in Fig. 7. For notational convenience, the sum of the long time average and doublyphase-resolved component will be denoted as

$$
\stackrel{\leftrightarrow}{\zeta}^{a p}\left(\phi_{a}, \phi_{h}\right)=\stackrel{\leftrightarrow}{\zeta}+\zeta^{a p}\left(\phi_{a}, \phi_{h}\right)
$$

Steinberg et al. [31] showed that the doubly-phase-resolved statistics, namely, $\stackrel{\leftrightarrow}{\zeta} \bar{c}\left(\phi_{a}, \phi_{h}\right)$, provide a good representation of the repeatable flow and flame configurations, where the flow is represented by $\zeta=\mathbf{u}$ and the flame is represented by the flame surface density (FSD) field, $\zeta=\Sigma$. The flame surface density describes the area of the reaction layers $\left(A_{f}\right)$ in a given volume $(\delta V)$ and is given by $\Sigma=\delta A_{f} / \delta V$. Here, the two-dimensional, doublyphase-resolved flame surface density field was calculated by dividing the measurement domain into $2 \times 2 \mathrm{~mm}$ cells and determining the doubly-phase-resolved mean reaction-layer length in each cell. A discussion of the statistical uncertainty in the doubly-phase-resolved analysis is given in Appendix A.

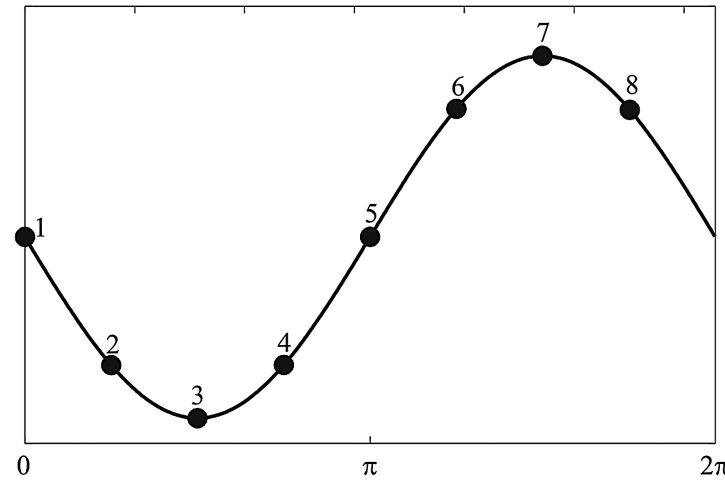

Fig. 7 Definition of phase angles for periodic analysis. 


\section{Oscillatory Flow and Flame Dynamics}

\section{A. Qualitative Analysis of HVC and Flame Behavior}

The above discussion illustrates some of the complex flow and flame processes occurring in this combustor. Understanding such processes is necessary in order to describe the fluid mechanical mechanisms affecting the thermoacoustic instability. However, before quantitatively analyzing these processes, it is informative to qualitatively observe their thermoacoustically coupled dynamics. To aid in this visualization, the doubly-phase-resolved statistics will be used to reconstruct the oscillatory flow and FSD behavior in three dimensions at each discrete phase angle over the thermoacoustic cycle. For a fixed $\phi_{a}$, the statistical variation of the flow and flame with changing $\phi_{h}$ represents different slices of the average 3-D fields at that $\phi_{a}$. The HVC phase can be thought of as representing the physical angle in the combustor through which each slice is acquired. For example, at $\phi_{a}=3$, the doubly-phase-resolved planar fields of $\stackrel{\leftrightarrow}{u}_{y}^{a p}\left(\phi_{a}=3, \phi_{h}=1 \ldots 8\right)$ represent eight different slices of the mean 3 -D axial velocity field at the minimum of the pressure cycle. The 3-D structure of the flow may be deduced by interpolating between these various slices. By performing this operation at each $\phi_{a}$, the dynamics of the 3-D flow and FSD fields over the thermoacoustic cycle can be visualized. If the reconstruction is taken such that the zero azimuthal angle is at a fixed $\phi_{h}\left(\phi_{h}=1\right.$ used here), the 3-D reconstructions are done in a $\mathrm{HVC}$-fixed reference frame. This frame rotates in the laboratory reference frame at $f_{h}$.

The planar three-component velocity measurements allow reconstruction of the full three-component three-dimensional

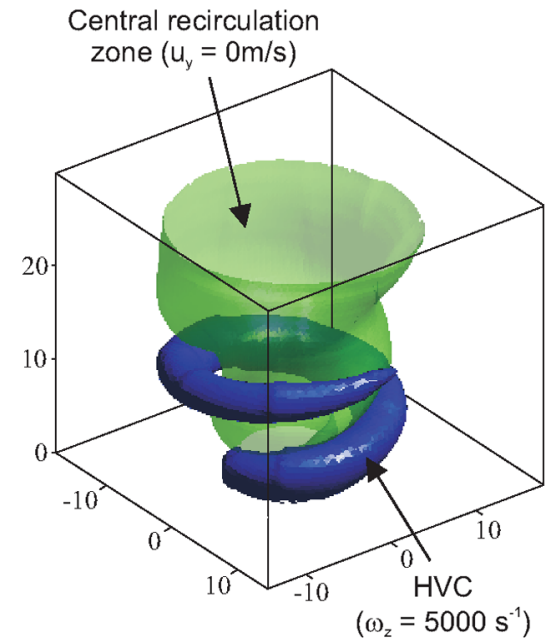

a) $\phi_{\mathrm{a}}=\mathbf{3}, p=p_{\min }$

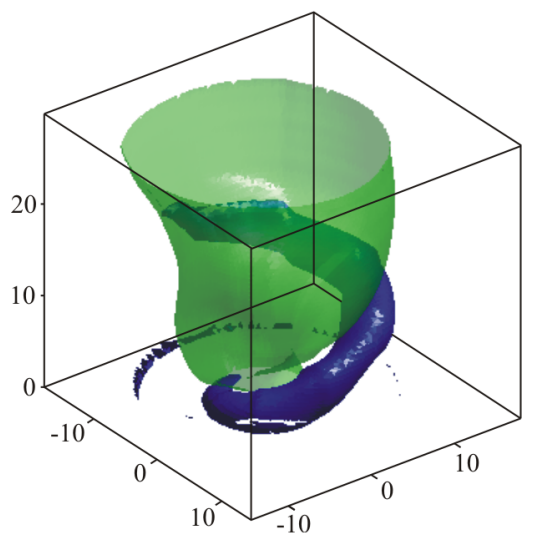

b) $\phi_{\mathrm{a}}=7, p=p_{\max }$

Fig. 8 Three-dimensional HVC $\left(\stackrel{\leftrightarrow}{\omega}_{z}^{a p}=\mathbf{5 0 0 0 ~ s} \mathrm{s}^{-1}\right)$ and CRZ $\left(\leftrightarrow_{u}^{a p}=0 \mathrm{~m} / \mathrm{s}\right)$ dynamics over an acoustic cycle in flame 2 from the doubly-phase-resolved mean vorticity and axial velocity fields. Axis units are in millimeters. velocity field, albeit with low resolution in the azimuthal direction. Because of this low azimuthal resolution, all velocity gradients are calculated only from the measured in-plane values (i.e., only $\omega_{z}$ ). For the flame surface density, the reconstructed fields are 3-D topographic maps of the planar $\stackrel{\leftrightarrow}{\Sigma}$ fp fields, not the three-dimensional FSD [42]. Such topographies are sufficient for examining the flame dynamics.

Figures $\underline{8}$ and 9 show the dynamics of the reconstructed 3-D HVC and CRZ at two phases of the thermoacoustic cycle for flame 2, which were determined from the doubly-phase-resolved statistics. The flame 2 flow structures are shown because the HVC intersected the measurement plane more times than in flame 3 and underwent larger thermoacoustically coupled dynamics than in flame 1 . The HVC is visualized in terms of a vorticity magnitude isosurface

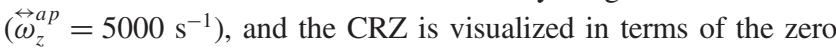
axial velocity isosurface $\left(\leftrightarrow_{y}^{a p}=0\right)$. Various other methods to visualize the HVC also were tested. These include the $\lambda_{2}$ criterion [43], the $\Delta$ criterion [44], and the swirling-strength criterion [45]. A detailed comparison of different methods for visualizing vortex cores is presented by Chakraborty et al. [46]. Although these methods provide a threshold-independent method of visualizing the HVC, their precise meaning can be ambiguous in the presence of the large density, species, and temperature gradients associated with reacting flows. Furthermore, the flow structures determined by all methods were qualitatively the same, exhibiting only minor differences in the boundaries and cross-sectional centroid positions. For ease of comparison with previous results, the above mentioned vorticity and axial velocity isosurfaces will be used to visualize the flow structures.

The thermoacoustically coupled HVC dynamics and their effect on the CRZ are clearly illustrated in these figures. At low combustion-chamber pressure $\left(\phi_{a}=3\right)$, the HVC is contracted axially and radially. Several spirals of the HVC were present in the PIV field of view and these spirals caused large-scale corrugations of the CRZ. When the HVC was extended at the maximum pressure $\left(\phi_{a}=7\right)$, fewer spirals were in the field of view and the CRZ was considerably less corrugated.

Steinberg et al. [31] showed that there were significant thermoacoustically coupled flame surface area oscillations associated with periodic reaction-layer corrugation at the scale of the HVC. That is, at some phase angles in the thermoacoustic cycle, the $\mathrm{HVC}$ interacted with the flame to create flame area, and at other phase angles it did not. Figure 10 shows the interaction of the HVC with the flame surface density field at two phase angles of the thermoacoustic cycle in flame 2. The FSD is represented by an isosurface at approximately half of the peak value, which well represents the shape of the overall 3-D field. The qualitative description of the relative flame and HVC shapes is insensitive to the particular isosurface value. Although velocity measurements only were taken in the region of the burner nozzle, it clearly can be seen that the HVC interacts with the flame throughout the combustor volume and that the degree of

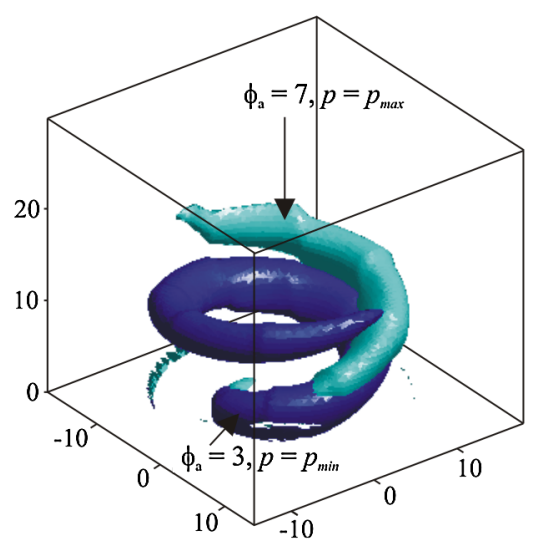

Fig. 9 Relative shapes of the HVCs $\left(\overleftrightarrow{\omega}_{z}^{a p}=5000 \mathrm{~s}^{-1}\right)$ in flame 2 at the minimum $\left(\phi_{a}=3\right)$ and maximum $\left(\phi_{a}=7\right)$ combustion-chamber pressure. Axis units are in millimeters. 


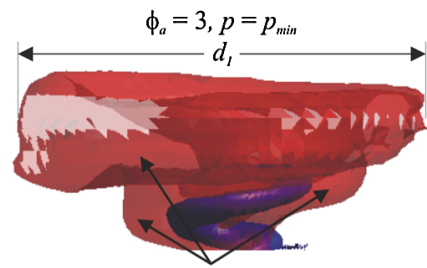

HVC induced flame corrugations

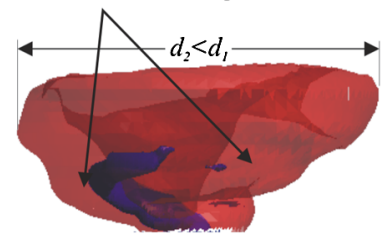

$\phi_{a}=7, p=p_{\text {max }}$

$-40 \mathrm{~mm} 40 \mathrm{~mm}$

Fig. 10 Measured interactions of the HVC $\left(\leftrightarrow_{\bar{\omega}}^{a p}=5000 \mathrm{~s}^{-1}\right)$ with the flame surface density field (representative isosurface of $\stackrel{\leftrightarrow}{\Sigma}^{a p}=0.12 \mathrm{~mm}^{-1}$ ) in flame 2 . The dynamic deformation of the HVC causes changing large-scale corrugation of the flame.

interaction changes as the HVC deforms over the thermoacoustic cycle. In this flame, the HVC was associated with greater flame corrugation when it is axially contracted.

In addition to illustrating these oscillating large-scale corrugations of the flame surface, Fig. 10 also shows that the flame undergoes radial dynamics over the thermoacoustic cycle. Figure 11a shows a flame surface density isosurface at twice the value of that shown in Fig. 10, indicating statistically the region of highest heat release, along with the HVC shape over the thermoacoustic cycle in flame 2. In the HVC-fixed frame that is produced by the doubly-phaseresolved analysis, the region of highest flame surface density rotates counter to the HVC motion and circumscribes the burner over the thermoacoustic cycle. In a laboratory fixed frame, the high-FSD region rotates in the direction of the HVC motion at the difference between the HVC and thermoacoustic frequencies. This process explains the radial motion of the heat-release centroid that was observed in the $\mathrm{OH}^{*}$ chemiluminescence measurements.

It previously has been observed that the asymmetry in the flame was associated with a deflection of the inflowing reactants caused by the proximity of a HVC branch downstream of the burner nozzle [31]. Since the HVC undergoes axial contraction and extension, the azimuthal locations at which the HVC is in proximity to the nozzle oscillated over the thermoacoustic cycle. This process can be further elucidated by Fig. 11b. In this figure, the arrows indicate the location, direction, and magnitude of local maxima in the radial velocity. It clearly can be seen that the local maxima are associated with the lower boundary of the HVC and that the azimuthal position of the highest-magnitude radial velocity changes with the HVC shape. Note that the HVCs at $\phi_{a}=1$ and 7 appear to have similar shapes but cause different radial velocity patterns. However, it will be shown in Sec. V.B that the lower portion of the HVC at these phase angles actually have quite different shapes; the lower portion is more axially contracted at $\phi_{a}=1$ and therefore causes a different radial velocity pattern.

\section{B. Quantification of HVC Behavior}

From the above discussion, it is clear that the axial deformation of the HVC over the thermoacoustic cycle greatly influences the oscillatory flame behavior. It is therefore beneficial to quantify the dynamics of this deformation. To do so, the locations at which the HVC spirals intersected the measurement plane were identified by local peak vorticity magnitudes in an alternating pattern of positive and negative $\stackrel{\leftrightarrow}{\omega}_{z}^{a p}$ extrema on each side of the burner axis in the axial direction. The shape of the HVC helix in all flames is shown in Fig. 12 at three phase angles of the thermoacoustic cycle. Once again, the axial deformation of the HVC is apparent. In all cases, the highest measured branch of the $\mathrm{HVC}$ at $\phi_{a}=3$ was relatively low, representing an overall contraction of the HVC. Conversely, at $\phi_{a}=7$ the highest branch was farther downstream than at $\phi_{a}=3$, representing an overall axial extension. The overall shapes of the HVC in flames 1 and 2 were similar, and the HVC in flame 3 was considerably flatter. Furthermore, the amplitude of the axial deformation can be seen to increase with the pressure oscillation amplitude from flame 1 to flame 3 .

These dynamics are further elucidated in Fig. 13, which shows the axial position of the HVC $\left(y_{h}\right)$ versus the angular position along the helix $\left(\theta_{h}\right)$ at different $\phi_{a}$ in each flame. The HVC undergoes similar dynamics in all flames. Starting at $\phi_{a}=1\left(p=\bar{p}\right.$ and $\left.\mathrm{d} p / \mathrm{d} \phi_{a}<0\right)$, the lower portion of the helix was axially contracted and the upper portion was extended. This is indicated by the low and high slopes of

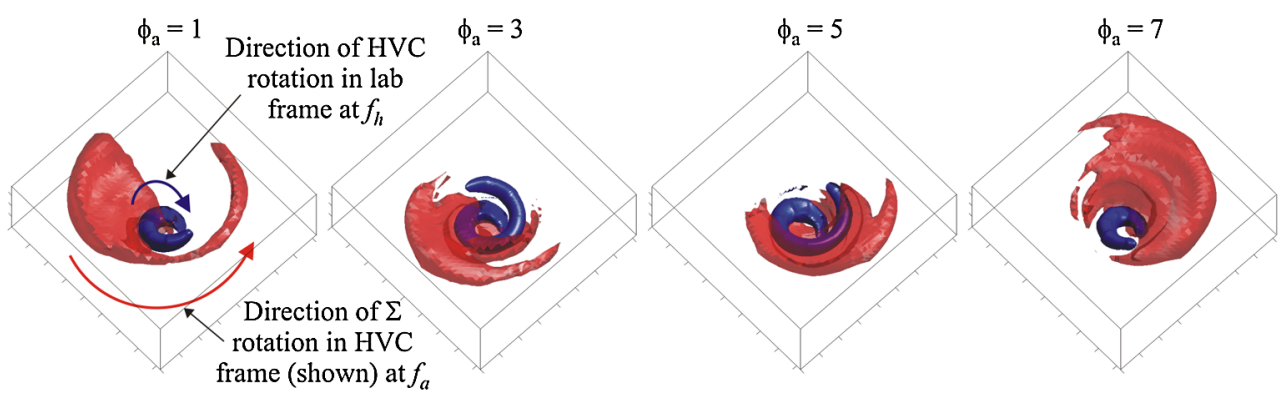

a) Flame surface density iso-surface $\left(\stackrel{\leftrightarrow}{\Sigma}{ }^{a p}=0.25 \mathrm{~mm}^{-1}\right.$, light $)$ representing statistically intense combustion with the HVC $\left(\stackrel{\leftrightarrow}{\omega}_{z}^{a p}=5000 \mathrm{~s}^{-1}\right.$, dark $)$

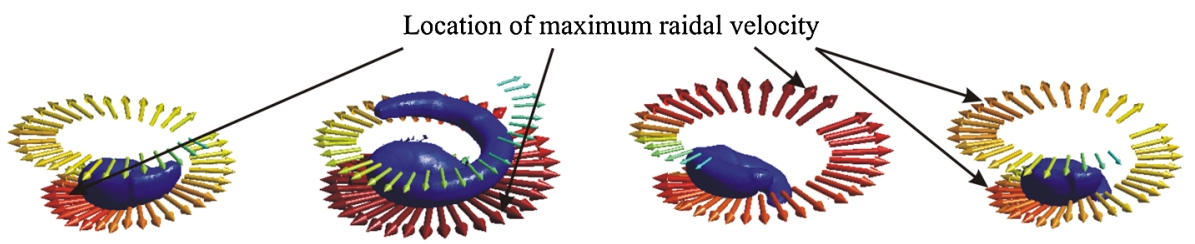

b) Relationship between the radial velocity field and HVC shape. Arrows are located at positions where the radial velocity is a local maximum. The length of the arrows represents the radial velocity magnitude

Fig. 11 Mechanism causing the heat release to circumscribe the combustor at $f_{q c}$. Images shown are for flame 2 and are in a HVC-fixed frame that rotates in the laboratory frame at $f_{h}$. 


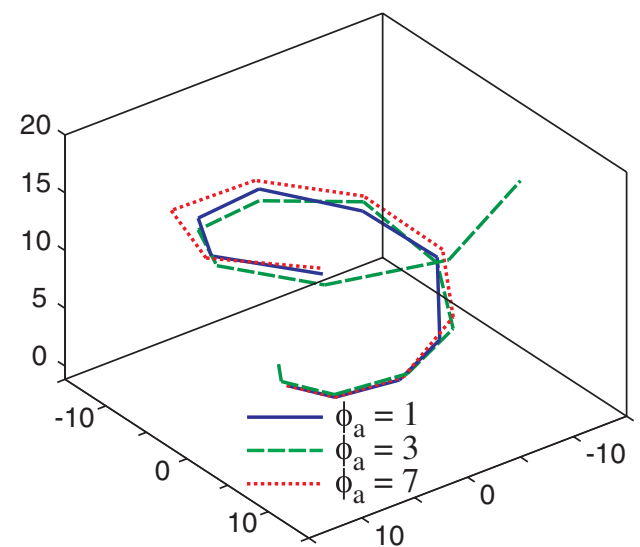

a) Flame 1

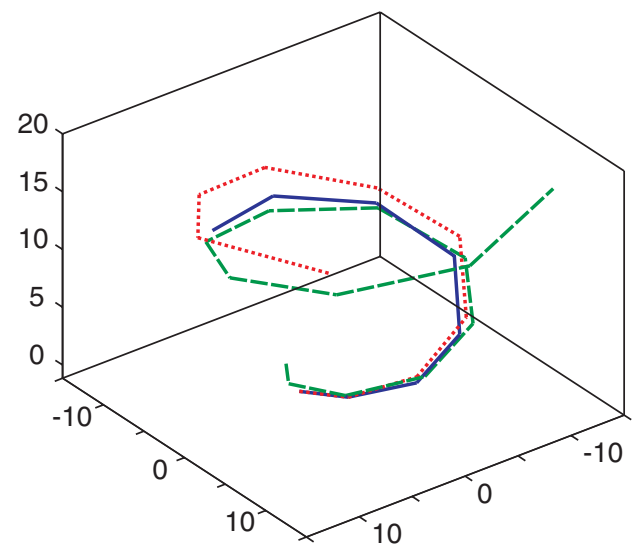

b) Flame 2

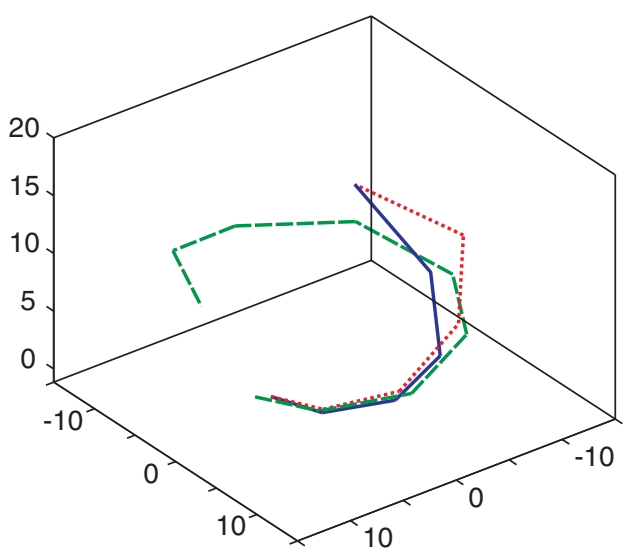

c) Flame 3

Fig. 12 Shape of the HVC helices over the thermoacoustic cycle in all flames. Axis units are in millimeters.

the $y_{h}$ curves at low and high $\theta_{h}$, respectively. As the combustionchamber pressure dropped $\left(\phi_{a}=3\right)$, the upper portion of the HVC axially contracted, resulting in the most compact helix in each flame. During the subsequent pressure rise, first the lower portion of the HVC axially extended $\left(\phi_{a}=5\right)$, followed by the upper portion $\left(\phi_{a}=7\right)$. At this latter pressure, the HVC was in its most axially extended configuration overall. This indicates that axial deformation of the HVC occurs as a wave, with different portions of the helix being displaced in different directions at different phases in the pressure cycle.

This also is demonstrated in Fig. 14, which shows the vertical displacement of particular points on the helix over the

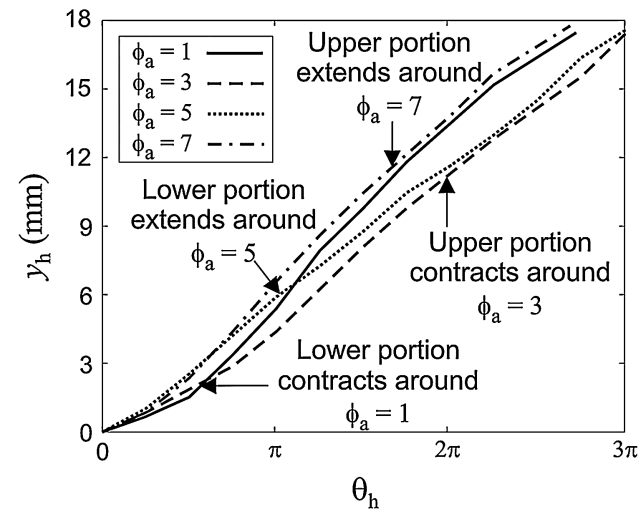

a) Flame 1

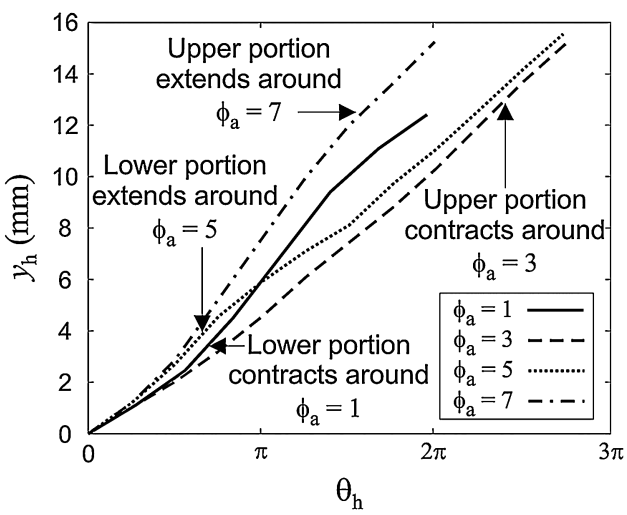

b) Flame 2

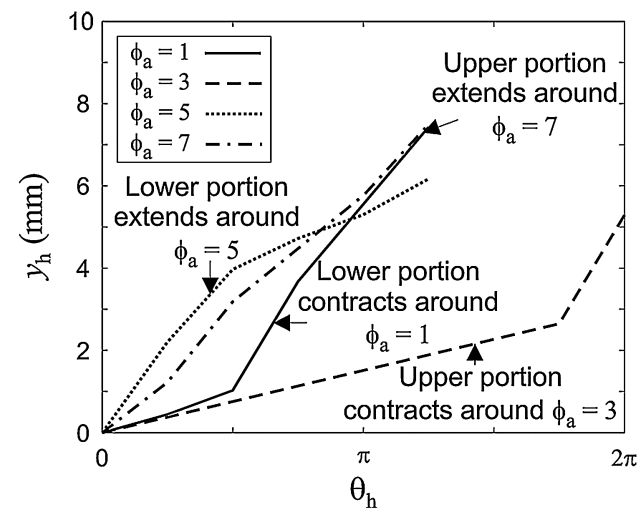

c) Flame 3

Fig. 13 Shape of the HVCs in terms of axial coordinate versus angle along the helix.

thermoacoustic cycle. In flame 1, the HVC was shaped such that it was in the PIV field of view for several spirals. Displacements are therefore shown at $\theta_{h}=\pi, 2 \pi$, and $3 \pi$. Fewer spirals were available for the other flames. As can be seen, HVC spirals at different downstream locations underwent axial displacement oscillations at different phases in the thermoacoustic cycle. In particular, the peak displacements of subsequent spirals in the axial direction tended to occur approximately $\pi / 4$ later in the thermoacoustic cycle. This lag corresponds well to the mean convective time between spirals, indicating that the $\mathrm{HVC}$ dynamics are driven by a convective process. The amplitude of the axial deformation also can be seen to increase with the amplitude of the thermoacoustic pulsations. However, the exact process that sets the phase shift and amplitude is not yet known. Further measurements with a larger field of view and over a larger parameter set will help uncover this mechanism.

\section{Periodic Flow/Flame Interactions}

The above analysis has demonstrated the complex dynamics of helical vortex cores in thermoacoustically oscillating swirl flames 


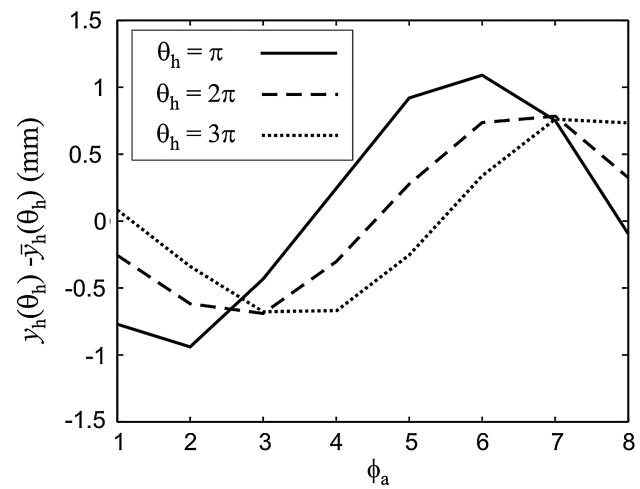

a) Flame 1

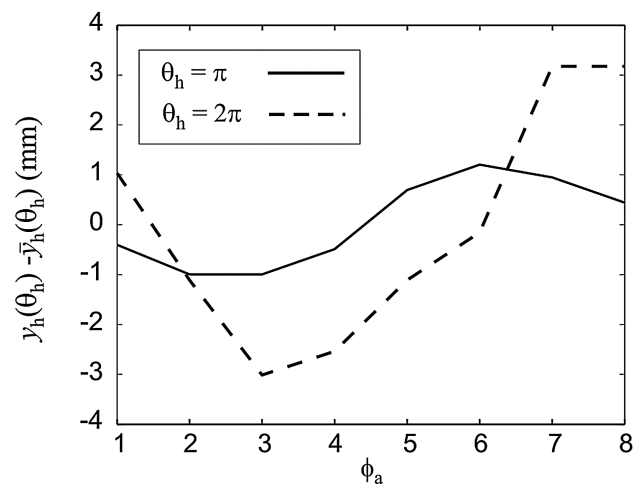

b) Flame 2

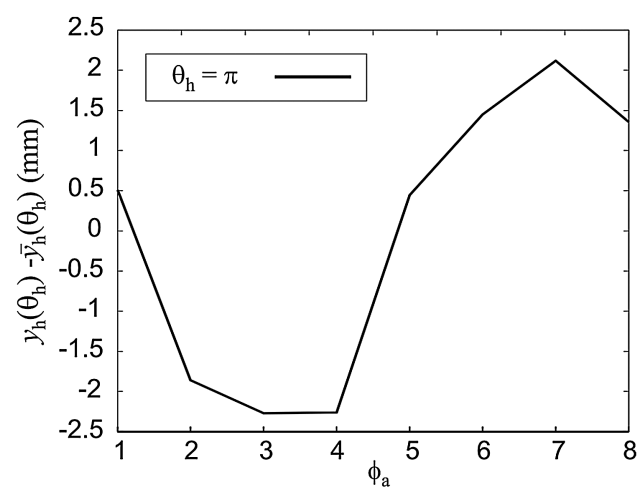

c) Flame 3

Fig. 14 Axial displacement of characteristic points along the HVC helices over the thermoacoustic cycle. and that these dynamics have a large effect on the flame surface. As the HVCs change shape over the thermoacoustic cycle, they can interact with the flame in a periodic manner, causing thermoacoustically coupled changes in the heat-release rate. The POD analysis showed that the HVCs were, by far, the most energetic flow structures. Hence, the periodic HVC/flame interactions may be very significant in determining the thermoacoustic state of the combustor.

To illustrate this further, the flow/flame interactions in the thermoacoustically stable (flame 1) and unstable (flame 3 ) cases will first be qualitatively compared. The details of the thermoacoustic coupling will then be discussed in Sec. VI. Figure 15 shows the reconstructed flame surface density field and $\mathrm{HVC}$ shape in flame 1 over the thermoacoustic cycle. Also shown are the mean combustor pressure, integrated $\mathrm{OH}^{*}$ chemiluminescence (heat release), total flame area, and reactant volume flux oscillations. To compliment these images, Fig. 16 shows typical instantaneous images of the flame topography and flowfield at select pressure phase angles. It is once again noted that the field of view for the PIV measurements was smaller than that of the OH-PLIF measurements. Hence, the HVC shape was directly measured only in the vicinity of the burner nozzle, but its influence can still be observed in the flame surface outside the PIV field of view. Also, this discussion will focus on heat-release oscillations driven by changes in the reactive surface area. The $\mathrm{OH}^{*}$ chemiluminescence measurements that provide the heat-release curves were line-of-sight integrated and therefore did not provide the spatial resolution necessary for the analysis. However, it can be seen that the flame area oscillations account for a large portion of the overall chemiluminescence oscillations.

At the peak of the pressure oscillation, the HVC was in its most axially extended configuration. When in this configuration, the HVC in flame 1 had a steeper angle than the flame surface relative to the burner exit plane; the majority of the HVC resided downstream of the flame in the burnt products. Both the doubly-phase-resolved 3-D reconstructions and instantaneous measurements show a relatively smooth flame. A single FSD corrugation can be seen in the lower portion of Fig. 15 at $\phi_{a}=7$, where the HVC crosses through the flame brush. In the instantaneous image (Fig. 16a), the flame segment that would be corrugated by the HVC in the lower-left region appears to be locally extinguished. As the combustion-chamber pressure decreases, the HVC axially contracts as described above. In doing so, it comes into contact with the flame. At the pressure minimum, $\phi_{a}=3$, the FSD field and instantaneous flame surface exhibit several large-scale corrugations, which increase the flame area and the overall heat-release rate. The 3-D corrugations are shown from a different view point in Fig. 17 and are clearly associated with the measured HVC shape. Hence, in this thermoacoustically quiet flame, the HVC appears to cause oscillations in the heat-release rate that are largely out of phase with the pressure oscillation. Also, the FSD field undergoes large-scale elongation due to the reactant flux oscillation.
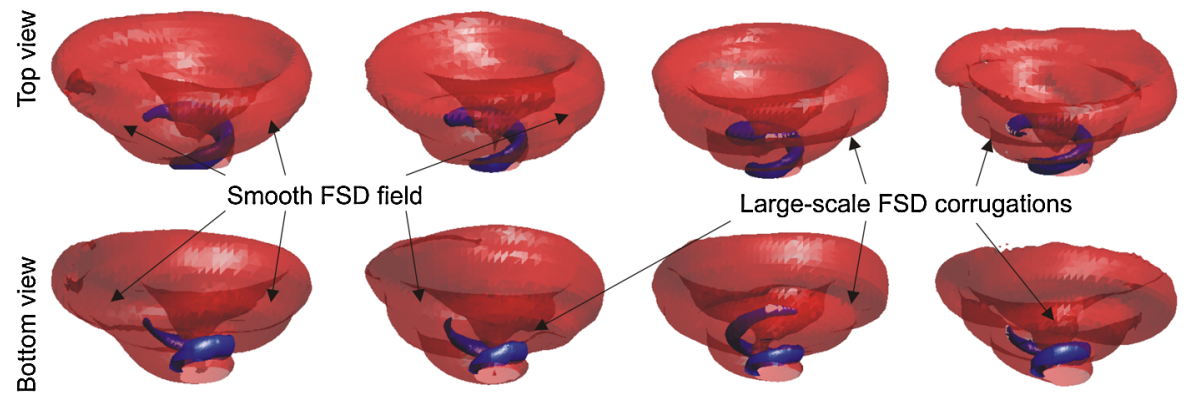

Large-scale FSD corrugations
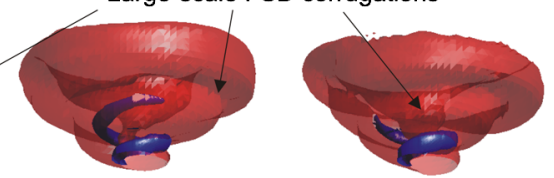

$\phi_{\mathrm{a}}=7$

$\phi_{\mathrm{a}}=1$

$\phi_{\mathrm{a}}=3$

$\phi_{\mathrm{a}}=5$

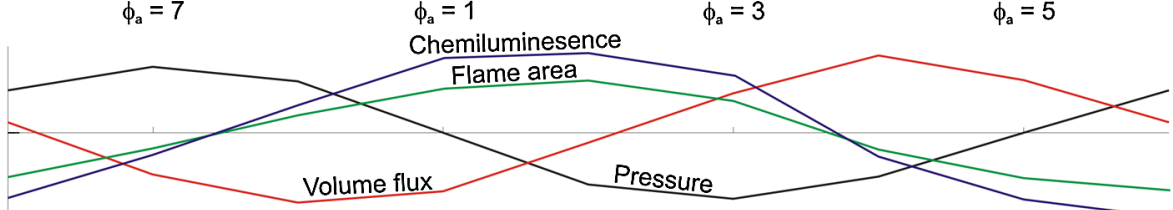

Fig. 15 Reconstructed HVC $\left(\stackrel{\leftrightarrow}{\omega}_{z}^{a p}=5000 \mathrm{~s}^{-1}\right)$ and flame surface density $\left(\stackrel{\leftrightarrow}{\Sigma}{ }^{a p}=0.12 \mathrm{~mm}^{-1}\right)$ for flame 1 over the thermoacoustic cycle. 
However this effect is quite small due to the low amplitude of the pressure oscillation.

A distinct difference in the $\mathrm{HVC} /$ flame interaction is observed in flame 3. Figure 18 shows the flame surface density fields, HVC shapes, and mean combustion-chamber oscillations for this flame, and Fig. 19 shows typical instantaneous measurements. A significant difference in the overall shape of the flame and HVC is apparent compared with flame 1 , with both features exhibiting a much lower slope relative to the burner exit plane. Like flame 1, the HVC underwent axial contraction and extension over the thermoacoustic cycle. Unlike flame 1, the HVC at the peak of the pressure cycle $\left(\phi_{a}=7\right)$ overlapped with the flame surface and caused several large-
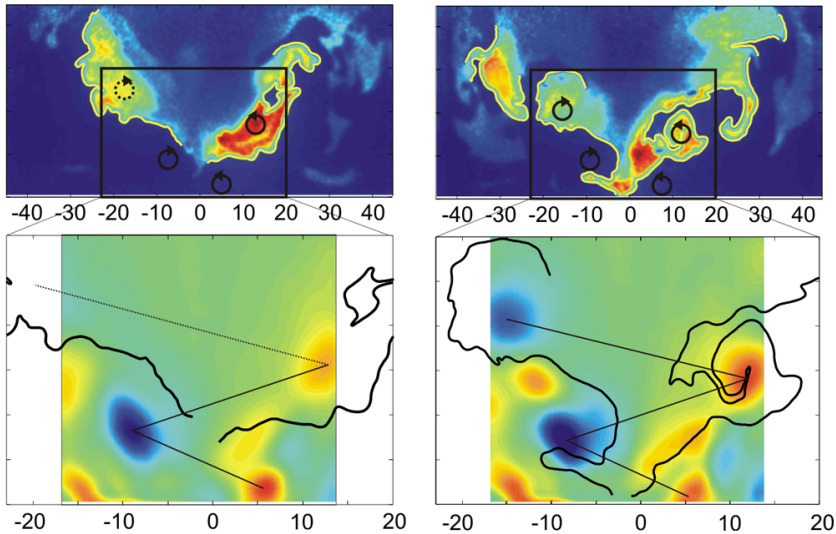

a) $\phi_{a}=7, p=p_{\max }$, few flame corrugations due to $\mathrm{HVC}$

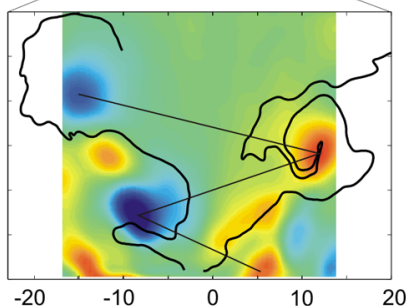

b) $\phi_{a}=3, p=p_{\text {min }}$, several large flame corrugations due to HVC

Fig. 16 Typical instantaneous measurements of the $\mathrm{OH}$ field, flame topography, and vorticity field at the minimum and maximum of the pressure oscillation in flame 1. $\omega_{z}$ between $-20,000 \mathrm{~s}^{-1}$ and $20,000 \mathrm{~s}^{-1}$, with the scale given in Fig. 5.

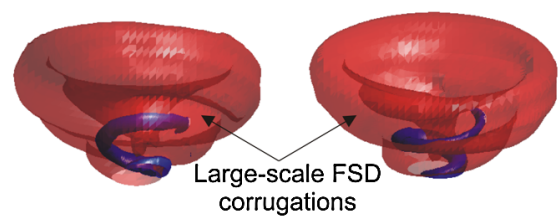

Fig. 17 Reconstructed HVC $\left(\stackrel{\leftrightarrow}{\omega_{z}^{a p}}=\mathbf{5 0 0 0} \mathrm{s}^{-1}\right)$ and flame surface density $\left(\stackrel{\leftrightarrow}{\Sigma}{ }^{a p}=0.12 \mathrm{~mm}^{-1}\right)$ for flame 1 at $\phi_{a}=3\left(p=p_{\min }\right)$ showing large-scale corrugations from a different viewpoint than in Fig. 15.
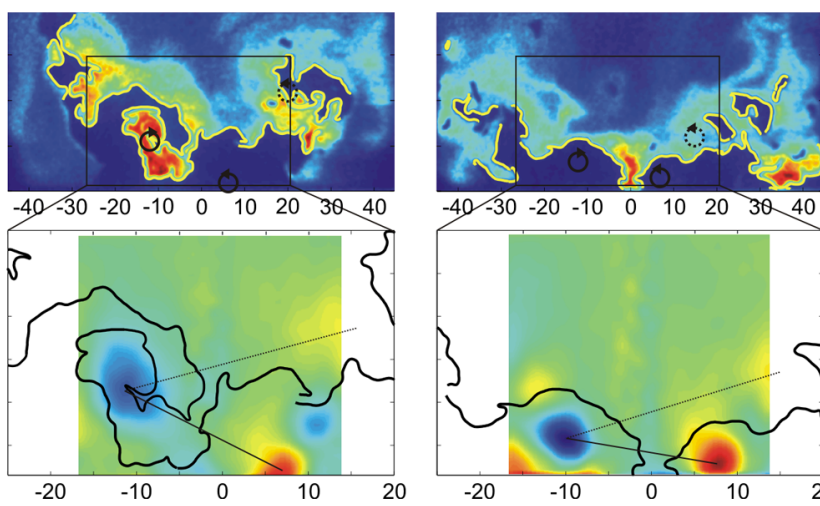

a) $\phi_{a}=7, p=p_{\max }$, the HVC is extended, interacts with the flame, and causes large flame corrugations

Fig. 19 Typical instantaneous measurements of the $\mathrm{OH}$ field, flame topography, and vorticity field at the minimum and maximum of the pressure oscillation in flame 3. $\omega_{z}$ between $-20,000 \mathrm{~s}^{-1}$ and $20,000 \mathrm{~s}^{-1}$, with the scale given in Fig. $\underline{5}$.

scale flame corrugations. As the pressure decreased, the HVC axially contracted to the extent that, at the pressure minimum $\left(\phi_{a}=3\right)$, it was below the flame surface. Figure 20 shows the relative positions of the HVC and FSD from a different view point than in Fig. 18. While in this shape, the HVC caused only a minor corrugation in the lower surface of the FSD field and the upper surface was smooth. Hence, the HVC in flame 3 caused increases in the flame area through wrinkling that were relatively in phase with the pressure oscillations. The FSD field also undergoes substantially greater radial elongation and contraction than was observed in flame 1 due to the larger reactant flux oscillations. Finally, the FSD fields are asymmetric and the location of the asymmetry rotates noticeably around the burner over the thermoacoustic cycle. Referring to the top-view images, the region of concentrated FSD rotates counterclockwise. This is the same phenomena that was described in Fig. 11. As opposed to flames 1 and 2 (Figs. 10 and 15), the asymmetry is associated with significant combustion in the outer recirculation zones (ORZs). Both the amount and azimuthal position of this ORZ combustion varies over the thermoacoustic cycle.

\section{Thermoacoustic Coupling}

A critical parameter that determines the amplitude of the heat release and acoustic oscillations is the net acoustic energy transfer $\Theta$,
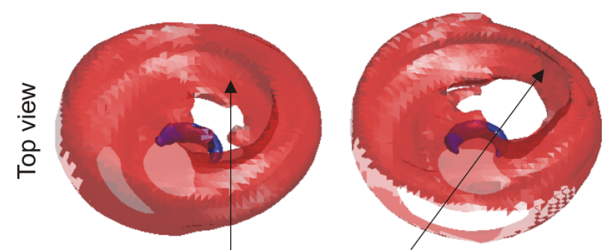

Large-scale FSD corrugations
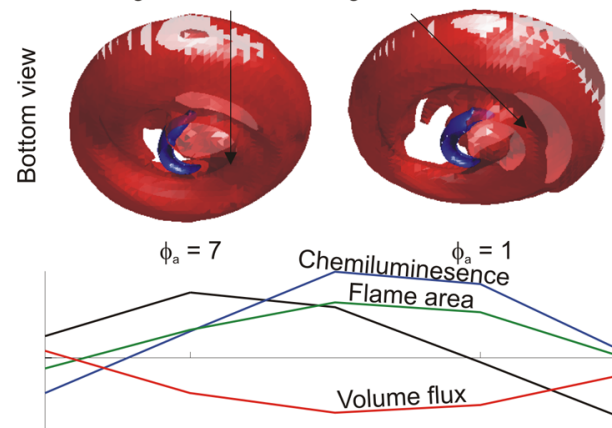
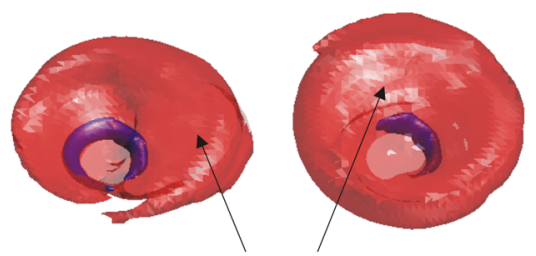

Smooth FSD field
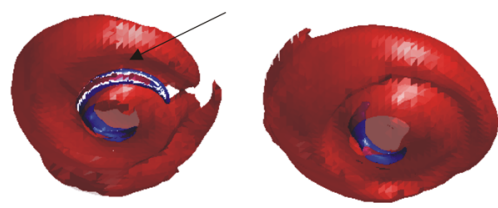

$\phi_{\mathrm{a}}=3$

$\phi_{\mathrm{a}}=5$

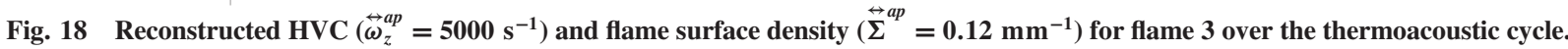


defined in Eq. (1). The nature of the local energy transfer, $\vartheta(\mathbf{x})$, is set by the phase shift, $\beta(\mathbf{x})$, between the pressure and heat-release oscillations. Local oscillations with $0^{\circ}<|\beta|<90^{\circ}$ produce positive energy transfer (destabilizing), and oscillations $90^{\circ}<|\beta|<180^{\circ}$ produce negative energy transfer (stabilizing). The magnitude of the local energy transfer is set both by the specific phase shift and the local oscillation amplitudes. As was seen in Fig. 2, the amplitude of the pressure oscillations was relatively uniform everywhere in the combustion chamber. The local acoustic energy transfer therefore depends primarily on the phase shift and the heat-release oscillation amplitude. Using the present measurements, only the flame surface area oscillations could be spatially resolved. However, comparing the flame area oscillations and chemiluminescence oscillations in Figs. $\underline{15}$ and $\underline{18}$ shows that the flame area oscillation is a good representation of the overall heat-release oscillation amplitude and phase. Therefore, the following discussion will consider the coupling of the pressure and flame area oscillations, with the overall $(\Psi)$ and local $(\psi)$ couplings defined as

$$
\Psi=\int_{V} \psi(\mathbf{x}) \mathrm{d} V=\int_{V} \int_{0}^{2 \pi} p^{\prime}\left(\phi_{a}\right) \stackrel{\leftrightarrow}{\Sigma}^{a p}\left(\mathbf{x}, \phi_{a}\right) \mathrm{d} \phi_{a} \mathrm{~d} \mathbf{x}
$$

In each cell used for the FSD calculation, the flame surface area oscillation was periodic (roughly sinusoidal) over the thermoacoustic cycle. The amplitude and thermoacoustic phase shift of the oscillation differed between cells. To illustrate the influence of the HVC on thermoacoustic coupling, $\beta(\mathbf{x})$ first was mapped by fitting sinusoidal curve to the local $\stackrel{\leftrightarrow}{\Sigma}^{a p}$ oscillation and determining its phase shift relative to the pressure oscillation. Figure 21 shows the three-dimensional reconstruction of $\beta(\mathbf{x})$ in flame 1 . The phase-shift distribution consisted of intertwined helical regions of in-phase and out-of-phase thermoacoustic oscillations. Both in-phase and out-ofphase regions could be found at almost all axial distances from the burner, except for far downstream. This once again confirms that the behavior of the HVC is very important to the thermoacoustic oscillations. In the absence of the HVC, the phase shift at a given axial location would be independent of the azimuthal location in the burner. Because of the HVC, however, it is not until the far downstream position that a relatively circular torus of in-phase oscillations occurred, which was associated with the large-scale elongation of the

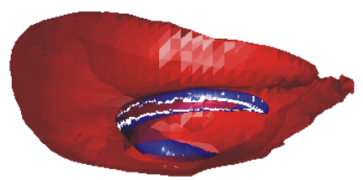

Fig. 20 Reconstructed HVC $\left(\stackrel{\leftrightarrow}{\omega}_{z}^{a p}=5000 \mathrm{~s}^{-1}\right)$ and flame surface density $\left({\stackrel{(}{\Sigma^{a}}}^{a p}=0.12 \mathrm{~mm}^{-1}\right)$ for flame 3 at $\phi_{a}=3\left(p=p_{\min }\right)$. The HVC was below the flame when axially contracted.

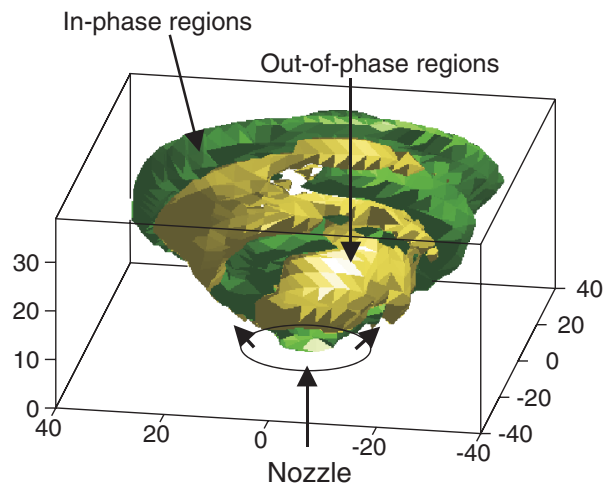

Fig. 21 Three-dimensional reconstructions of the combustor regions in which flame surface area oscillations were in phase $\left(0^{\circ}<|\beta|<90^{\circ}\right.$, light $)$ and out of phase $\left(90^{\circ}<|\beta|<180^{\circ}\right.$, dark) with the pressure oscillations in flame 1. Axis units are in millimeters. flame due to the oscillating reactant flux. It is interesting to note that the combustor volumes containing in-phase and out-of-phase thermoacoustic oscillations are similar in this quiet flame.

To fully understand the thermoacoustic coupling, both the details of the local phase shift and the magnitude of the heat-release oscillations must therefore be considered. This can be done by directly computing $\psi$ from the doubly-phase-resolved flame area oscillations using Eq. (4). Figure 22 shows positive and negative isosurfaces of $\psi$ at one-fourth of the maximum computed value in each flame. The viewpoints were chosen to highlight the topographic features and therefore change between flames. In flame 1 , immediately downstream of the burner exit were intertwined helical regions of positive and negative coupling caused by the HVC/flame interaction. Note the large helical region of negative coupling that wraps fully around the burner. This region is caused by the increased flame corrugations as the HVC axially contracts near the minimum of the pressure cycle. Hence, in this flame, the dynamic HVC/flame interaction caused increased flame surface area (heat release) at decreased combustion-chamber pressure and, therefore, negative thermoacoustic coupling that helped to stabilize oscillations. Further downstream was a toroidal region of negative coupling caused by the periodic elongation of the flame.

From various prior figures, it can be seen that the FSD fields and HVCs in flames 1 and 2 had similar shapes. Likewise, the major features of the thermoacoustic coupling in these flames were similar. That is, flame 2 contained intertwined helical regions of positive and negative coupling downstream of the burner exit. However, the region of positive coupling was much larger, and the region of negative coupling was restricted to a small volume near the burner axis. The positively coupled helical region in flame 2 had a flatter angle relative to the burner exit plane than in flame 1 and extended to a greater distance downstream. This downstream branch interfered with the negatively coupled toroidal region that was seen in flame 1 , and the corresponding region in flame 2 was restricted to one side of the burner. It is therefore apparent that the HVC dynamics in this flame resulted in more positive thermoacoustic coupling and helped drive the oscillations. The change in the thermoacoustic coupling relative to flame 1 is caused by a slight narrowing of the flame angle, which brings the flame into contact with the HVC over the entire thermoacoustic cycle (as opposed to only at the pressure minimum in flame 1). Hence, it is clear that the opening angles of the HVC and flame are very important in determining the thermoacoustic coupling.

The importance of the HVC and flame shapes is further emphasized in flame 3. It was previously observed that the FSD field and HVC in flame 3 had considerably different shapes than the other flames; both were flatter and there was considerable combustion in the outer recirculation zones. The thermoacoustic coupling field in this flame therefore has some different features than the other flames. However, the intertwined helical regions of positive and negative coupling still existed. These regions occurred as alternating pairs of negative and positive coupling, beginning with a small negative region near the nozzle exit and ending with a large positive region downstream. The positively coupled helical region was considerably larger than the negative region, which is related to an increase in the overall flame corrugation when the HVC is in its axially extended configuration as previously described. Two additional regions also are observed, a large positively coupled region in the outer recirculation zone (bottom corner of the combustor) and a smaller negatively coupled region in the opposite corner. Flame 3 was the only case in which there was combustion in the outer recirculation zone, which was related to a large radial deflection of incoming reactants by the compact HVC [31]. As shown in Fig. 18, the amount and position of the ORZ combustion varied periodically over the thermoacoustic cycle, as the reactant flux and HVC shapes oscillated. The net consequence of the radially compact HVC causing ORZ combustion, the oscillating reactant flux causing varying amounts of ORZ combustion, and the changing azimuthal position of the maximum radial velocity caused by the axial HVC dynamics, were the larger positive and smaller negative regions of thermoacoustic coupling. 


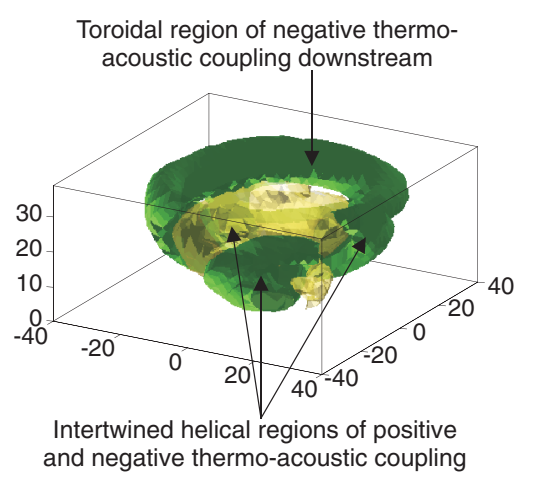

a) Flame 1, bottom view, $\psi= \pm 450 \mathrm{~Pa} / \mathrm{m}$

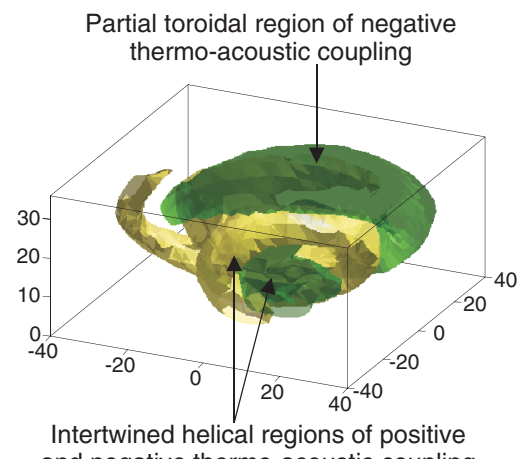

and negative thermo-acoustic coupling

b) Flame 2, bottom view, $\psi= \pm 1300 \mathrm{~Pa} / \mathrm{m}$

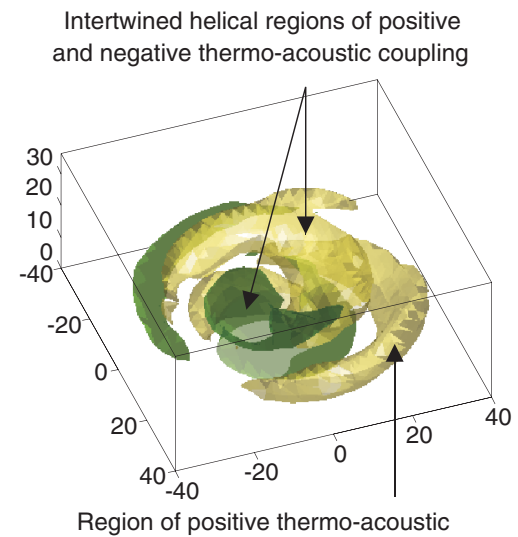

coupling in outer recirculation zone

c) Flame 3, top view, $\psi= \pm 2200 \mathrm{~Pa} / \mathrm{m}$

Fig. 22 Three-dimensional isosurfaces of the thermoacoustic coupling. Dark isosurfaces are negative $\psi$ and indicates the regions in which energy is removed from the acoustic field. Light isosurfaces are positive $\psi$ and indicates the regions in which energy is added from the acoustic field. Axis units are in millimeters.

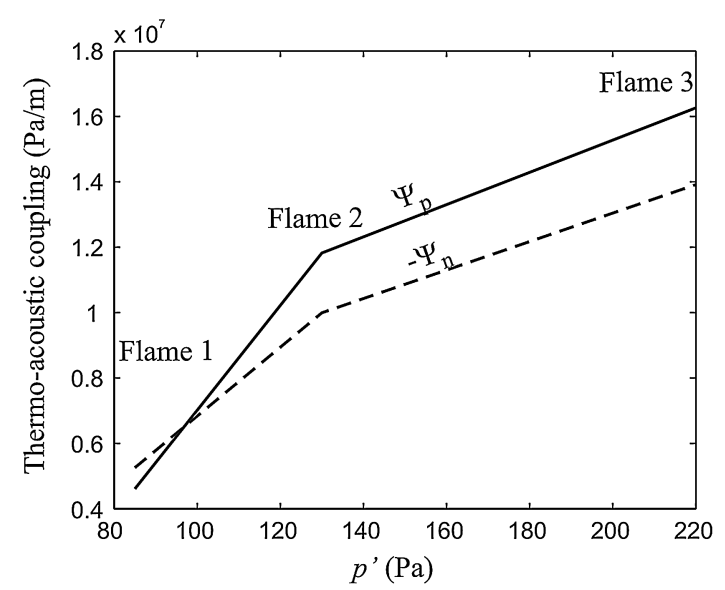

Fig. 23 Total positive and negative thermoacoustic coupling in each flame.

From these observations, it appears that an optimal flow/flame configuration is one in which the HVC has a slightly steeper angle than the flame relative to the burner exit plane. In such a configuration, the axial dynamics of the HVC causes increased flame area as the combustion-chamber pressure decreases. This results in a region of negative thermoacoustic coupling that can act as a natural damper to oscillations.

Figure 23 shows the net positive $\left(\Psi_{p}\right)$ and negative $\left(\Psi_{n}\right)$ thermoacoustic coupling in all flames versus the magnitude of the thermoacoustic pulsations. These were computed by performing the integral in Eq. (4) over the regions in which $\psi(\mathbf{x})>0$ and $\psi(\mathbf{x})<0$.
In the quiet flame 1 , the total negative coupling was slightly greater than the positive coupling. Moving to the louder flames 2 and 3, the positive coupling increased at a greater rate than the negative coupling, and the net thermoacoustic energy transfer was positive.

\section{Conclusions}

The thermoacoustic coupling caused by dynamic flow/flame interactions was investigated in a gas-turbine model combustor through analysis of high-repetition-rate laser diagnostics. Three fuellean, swirl-stabilized flames were investigated, each of which underwent different amplitude thermoacoustic pulsations and had flowfields dominated by a helical vortex core. By resolving the measurement sequence with respect to both the phase in the thermoacoustic cycle and the azimuthal position of the helical vortex core, the repeatable oscillatory processes were reconstructed in three dimensions. This allowed identification of the thermoacoustically coupled flow and flame dynamics, their interactions, and how these interactions affected the thermoacoustic phase-relationship and energy transfer.

Under all conditions, it was found that the helical vortex cores underwent axial deformation over the thermoacoustic cycle. At the minimum combustion-chamber pressure, the helices were in their most axially contracted configurations, and at the maximum chamber pressure they were in their most extended configurations. However, the displacement of particular spirals occurred at different points in the thermoacoustic cycle, and the axial deformation appeared to be associated with a convective wave. The amplitude of the deformation increased with the amplitude of the thermoacoustic oscillations.

The periodic deformation of the helical vortices caused them to interact with the flame in an oscillatory manner over the 
thermoacoustic cycle. Depending on the relative shapes of the flame and the helices in their axially extended and contracted configurations, these interactions caused oscillations in the flame surface area at different thermoacoustic phase angles. Hence, this behavior influenced the thermoacoustic state of the combustor.

To investigate this further, the local thermoacoustic coupling was determined in three dimensions throughout the combustor volume. In all cases, intertwined regions of positive and negative coupling occurred near the burner nozzle due to the helical vortices. In the quietest flame, the helical vortex created a large region of negative coupling that helped damp the thermoacoustic oscillations. In the moderately louder flame, the shapes of the helix and flame were such that there was a large helical region of positive thermoacoustic coupling that contributed energy to the thermoacoustic pulsations. In the loudest flame, positive thermoacoustic coupling occurred in both a large helical region and in the outer recirculation zone.

From this analysis, it is clear that the presence, shape, and dynamics of large-scale coherent vortical structures have a significant impact on the thermoacoustic state of combustors. Furthermore, the HVC dynamics can either damp or drive the instability, depending on the relative shape of the HVC and flame. The optimal configuration in this burner appears to be one in which the HVC has a slightly steeper angle than the flame relative to the burner exit plane. In this configuration, the HVC causes increased flame area at the minimum of the pressure fluctuation (when it is axially contracted), thus removing energy from the thermoacoustic oscillations.

It is therefore necessary to systemically study flame and flow structures in these swirl flames, in order to provide better predictive capabilities of their behavior. It appears possible that, if robustly understood and properly tuned, helical vortex cores may provide an effective means of passively extracting energy from thermoacoustic pulsations. Furthermore, this study has demonstrated an analytical method of spatially mapping thermoacoustic coupling in complex and practically relevant flowfields. The application of this technique to more industrial-like conditions will be performed in the future.

\section{Appendix A: Uncertainty Analysis}

The fundamental source of uncertainty in the velocity measurements is inaccuracies in the instantaneous fields computed from the PIV data. Based on a \pm 0.1 pixel uncertainty of the crosscorrelation peak-finding algorithm, the random uncertainty of the PIV measurements was estimated in Boxx et al. [29] to be approximately $5 \%$.

The accuracy of the doubly-phase-resolved mean fields in representing the repeatable flow dynamics is described by the standard deviation of the doubly-phase-resolved instantaneous measurements about the mean and the number of measurements used to compute the mean. Typically, about 125 instantaneous measurements were used at each of the 64 discrete phase-angle combinations. Integrated over the entire field of view, the average uncertainty in the mean fields was around $20 \%$. However, in locations with high-velocity gradients, this could be as high as approximately $50 \%$.

There are two major contributors to the variance in the velocity fields. First, the flow is not perfectly oscillatory, but exhibits cycle-tocycle variability and stochastic turbulence. Second, the continuous acoustic and HVC signals were sorted into eight discrete phase angles. Hence, any nominal phase angle at which the statistics are reported actually contains data over a two-dimensional band, including $\phi_{\text {nominal }}-\pi / 8$ to $\phi_{\text {nominal }}+\pi / 8$. Considering a particular slice $\left(\phi_{h}\right)$ of the flow, if the system were perfectly oscillatory, the variance in the mean due averaging the signals over the thermoacoustic bandwidth $(l=\pi / 4)$ is

$$
E\left(\phi_{a}\right)=\frac{1}{l}\left\{\int_{-l / 2}^{l / 2}\left[\sin \left(\phi_{a}+\zeta\right)-\sin \left(\phi_{a}\right)\right]^{2} \mathrm{~d} \zeta\right\}^{1 / 2}
$$

This function is plotted in Fig. A1 and reaches maxima at $\phi_{a}=0$ and $\pi$, where the sin function has its greatest slopes. At these points in the thermoacoustic cycle, the variance in the measured mean would be $25 \%$, regardless of any nonoscillatory aspects of the flow. Also plotted

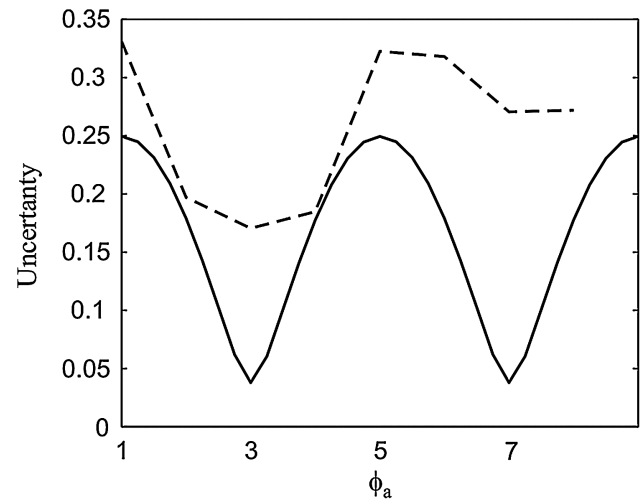

Fig. A1 Theoretical uncertainty due to discretization (Eq. (A1), (solid line) and measured uncertainty in the axial velocity over the thermoacoustic cycle in flame 3 (dashed line).

in this figure is the uncertainty in the measured doubly-phase-resolved mean axial velocity over the thermoacoustic cycle, averaged across the field of view at $\phi_{h}=1$ in flame 3 . This is the combination of parameters which had the greatest uncertainty. As can be seen, the uncertainty in the measurements also oscillated over the thermoacoustic cycle and had similar peaks to the theoretical curve. As expected, the measured uncertainty was always greater than the variance purely due to discretization, generally being about $10-15 \%$ higher. Improvements to the statistical convergence could therefore be accomplished both by increasing the number of discretization points and increasing the number of measurements at each point. Future measurements will attempt to employ approximately 20,000 planar measurements distributed across 144 phase-angle combinations.

Uncertainty in the reported flame surface density arises from the same discretization process as in the velocity fields, and also from the use of the OH-PLIF images to map the flame surface. Flame surface mapping from $\mathrm{OH}$ gradients is quite robust and the results presented are independent of any threshold values used during processing (over a reasonable range).

Calculation of the thermoacoustic coupling involves the integral of the product of two statistical quantities, the pressure and flame surface area. The pressure oscillation was quite regular in all cases and the majority of the uncertainty arose from discretization. To obtain an upper bound on the $\psi$ uncertainty, the integral in Eq. (4) was calculated using the measured signals, but displacing each point in the pressure oscillation upwards or downwards by $25 \%$ of its particular amplitude and each point in the flame area oscillation in the opposite direction by $35 \%$. In cases where the two signals were highly in phase or out of phase, the change in the computed coupling could be as large as about $25 \%$. Note that this value only matches the lower displacement amplitude because, inevitably, there was some cancellation of the noise at different phase angles of the cycle. In cases where the two signals were approximately $\pi / 2$ out of phase, the self-cancellation of the noise was greater and only about $15 \%$ uncertainty was observed.

\section{Acknowledgments}

The financial support within the DLR, German Aerospace Center, project Instationäre Verbrennung - Thermoakustische Schwingungen (IVTAS) is acknowledged. C. Carter acknowledges the support of the U.S. Air Force Office of Scientific Research Windows on Europe Program.

\section{References}

[1] Correa, S., "A Review of $\mathrm{NO}_{\mathrm{x}}$ Formation Under Gasturbine Combustion Conditions," Combustion Science and Technology, Vol. 87, 1993, pp. 329-362. doi:10.1080/00102209208947221

[2] Correa, S. M., "Power Generation and Aeropropulsion Gas Turbines: From Combustion Science to Combustion Technology," Symposium 
(International) on Combustion, Vol. 27, 1998, pp. 1793-1807. doi:10.1016/S0082-0784(98)80021-0

[3] Lefebvre, A., Gas Turbine Combustion, Taylor and Francis, New York, 1999.

[4] Lieuwen, T., and Yang, V. (eds.), Combustion Instabilities in Gas Turbine Engines: Operational Experience, Fundamental Mechanisms, and Modeling, AIAA, Reston, VA, 2006.

[5] Docquier, N., and Candel, S., "Combustion Control and Sensors: A Review," Progress in Energy and Combustion Science, Vol. 28, 2002, pp. $107-150$ doi:10.1016/S0360-1285(01)00009-0

[6] Emiris, I., and Whitelaw, J. H., "Control of Combustion Oscillations," Combustion Science and Technology, Vol. 175, No. 1, 2003, pp. 157-184. doi: $10.1080 / 00102200302363$

[7] Dowling, A. P., and Morgans, A. S., "Feedback Control of Combustion Oscillations," Annual Review of Fluid Mechanics, Vol. 37, 2005, pp. 151-182. doi:10.1146/annurev.fluid.36.050802.122038

[8] Paschereit, C. O., and Gutmark, E., "Active Control of Combustion Instabilities in Gas Turbine Burners," ASME Turbo Expo, Paper GT2006-90186, 2006, pp. 161-171.

[9] Candel, S. M., "Combustion Dynamics and Control: Progress and Challenges," Proceedings of the Combustion Institute, Vol. 29, 2002, pp. $1-28$. doi:10.1016/S1540-7489(02)80007-4

[10] Syred, N., "A Review of Oscillation Mechanisms and the Role of the Precessing Vortex Core (PVC) in Swirl Combustion Systems," Progress in Energy and Combustion Science, Vol. 32, 2006, pp. 93-161. doi:10.1016/j.pecs.2005.10.002

[11] Huang, Y., and Yang, V., "Dynamics and Stability of Lean-Premixed Swirl-Stabilized Combustion," Progress in Energy and Combustion Science, Vol. 35, 2009, pp. 293-365. doi:10.1016/j.pecs.2009.01.002

[12] Thumuluru, S. K., and Lieuwen, T., "Characterization of Acoustically Forced Swirl Flame Dynamics," Proceedings of the Combustion Institute, Vol. 32, 2009, pp. 2893-2900. doi:10.1016/j.proci.2008.05.037

[13] Roux, S., Lartigue, G., Poinsot, T., Meier, U., and Bérat, C., "Studies of Mean and Unsteady Flow in a Swirled Combustor Using Experiments, Acoustic Analysis and Large Eddy Simulations," Combustion and Flame, Vol. 141, 2005, pp. 40-54. doi:10.1016/j.combustflame.2004.12.007

[14] Weigand, P., Meier, W., Duan, X. R., Stricker, W., and Aigner, M., "Investigations of Swirl Flames in a Gas Turbine Model Combustor I. Flow Field, Structures, Temperature, and Species Distributions," Combustion and Flame, Vol. 144, 2006, pp. 205-224. doi:10.1016/j.combustflame.2005.07.010

[15] Paschereit, C. O., Gutmark, E., and Weisenstein, W., "Coherent Structures in Swirling Flows and Their Role in Acoustic Combustion Control," Physics of Fluids, Vol. 11, 1999, pp. 2667-2678. doi:10.1063/1.870128

[16] Wang, P., Baia, X. S., Wessman, M., and Klingmann, J., "Large Eddy Simulation and Experimental Studies of a Confined Turbulent Swirling Flow," Physics of Fluids, Vol. 16, 2004, pp. 3306-3324. doi:10.1063/1.1769420

[17] Fernandes, E. C., Heitor, M. V., and Shtork, S. I., "An Analysis of Unsteady Highly Turbulent Swirling Flow in a Model Vortex Combustor," Experiments in Fluids, Vol. 40, 2005, pp. 177-187. doi:10.1007/s00348-005-0034-4

[18] Cala, C. E., Fernandes, E. C., Heitor, M. V., and Shtork, S. I., "Coherent Structures in Unsteady Swirling Jet Flow," Experiments in Fluids, Vol. 40, 2006, pp. 267-276. doi: $10.1007 / \mathrm{s} 00348-005-0066-9$

[19] Wegner, B., Maltsev, A., Schneider, C., Sadiki, A., Dreizler, A., and Janicka, J., "Assessment of Unsteady RANS in Predicting Swirl Flow Instability Based on LES and Experiments," International Journal of Heat and Fluid Flow, Vol. 25, 2004, pp. 528-536. doi:10.1016/j.ijheatfluidflow.2004.02.019

[20] Garcia-Villalba, M., Fröhlich, J., and Rodi, W., "Numerical Simulations of Isothermal Flow in a Swirl Burner," Journal of Engineering for Gas Turbines and Power, Vol. 129, 2007, pp. 377-386. doi: $10.1115 / 1.2364198$

[21] Martinelli, F., Olivani, A., and Coghe, A., "Experimental Analysis of the Precessing Vortex Core in a Free Swirling Jet," Experiments in Fluids, Vol. 42, 2007, pp. 827-839. doi:10.1007/s00348-006-0230-x

[22] Wang, S., Yang, V., Hsiao, G., Hsieh, S.-Y., and Mongia, H. C., "LargeEddy Simulations of Gas-Turbine Swirl Injector Flow Dynamics,"
Journal of Fluid Mechanics, Vol. 583, 2007, pp. 99-122. doi: $10.1017 / \mathrm{S} 0022112007006155$

[23] Lacarelle, A., Faustmann, T., Greenblatt, D., Paschereit, C. O., Lehmann, O., Luchtenburg, D. M., and Noack, B. R., "Spatiotemporal Characterization of a Conical Swirler Flow Field Under Strong Forcing," Journal of Engineering for Gas Turbines and Power, Vol. 131, 2009, Paper 031504. doi:10.1115/1.2982139

[24] Iudiciani, P., and Duwig, C., "Large Eddy Simulation of the Sensitivity of Vortex Breakdown and Flame Stabilisation to Axial Forcing," Flow, Turbulence and Combustion, Vol. 86, 2011, pp. 639-666. doi:10.1007/s10494-011-9327-2

[25] Duan, X. R., Meier, W., Weigand, P., and Lehmann, B., "PhaseResolved Laser Raman Scattering and Laser Doppler Velocimetry Applied to Periodic Instabilities in a Gas Turbine Model Combustor," Applied Physics B (Lasers and Optics), Vol. 80, 2005, pp. 389-396. doi: $10.1007 / \mathrm{s} 00340-004-1722-0$

[26] Meier, W., Duan, X., and Weigand, P., "Investigations of Swirl Flames in a Gas Turbine Model Combustor II. Turbulence-Chemistry Interactions," Combustion and Flame, Vol. 144, 2006, pp. 225-236. doi:10.1016/j.combustflame.2005.07.009

[27] Sadanandan, R., Stöhr, M., and Meier, W., "Simultaneous OH-PLIF and PIV Measurements in a Gas Turbine Model Combustor," Applied Physics B (Lasers and Optics), Vol. 90, 2008, pp. 609-618. doi:10.1007/s00340-007-2928-8

[28] Stöhr, M., Sadanandan, R., and Meier, W., "Experimental Study of Unsteady Flame Structures of an Oscillating Swirl Flame in a Gas Turbine Model Combustor," Proceedings of the Combustion Institute, Vol. 32, 2009, pp. 2925-2932. doi:10.1016/i.proci.2008.05.086

[29] Boxx, I., Stöhr, M., Carter, C., and Meier, W., "Temporally resolved planar measurements of transient phenomena in a partially pre-mixed swirl flame in a gas turbine model combustor," Combustion and Flame, Vol. 157, 2010, pp. 1510-1525 doi:10.1016/j.combustflame.2009.12.015

[30] Stöhr, M., Boxx, I., Carter, C. D., and Meier, W., "Dynamics of Lean Blowout of a Swirl-Stabilized Flame in a Gas Turbine Model Combustor," Proceedings of the Combustion Institute, Vol. 33, 2011, pp. 1663-1672. doi:10.1016/j.proci.2010.06.134

[31] Steinberg, A. M., Boxx, I., Stöhr, M., Carter, C. D., and Meier, W., "Flow-Flame Interactions Causing Acoustically Coupled Heat Release Fluctuations in a Thermoacoustically Unstable Gas Turbine Model Combustor," Combustion and Flame, Vol. 157, 2010, pp. 2250-2266. doi:10.1016/j.combustflame.2010.07.011

[32] Steinberg, A. M., Boxx, I., Stöhr, M., Carter, C. D., and Meier, W. "Analysis of Flow/Flame Interactions in a Gas Turbine Model Combustor Under Thermoacoustically Stable and Unstable Conditions," 46th AIAA/ASME/SAE/ASEE Joint Propulsion Conference \& Exhibit, AIAA Paper 2010-7152, 2010.

[33] Najm, H., Paul, P., Mueller, C., and Wyckoff, P., "On the Adequacy of Certain Experimental Observables as Measurements of Flame Burning Rate," Combustion and Flame, Vol. 113, 1998, pp. 312-332. doi:10.1016/S0010-2180(97)00209-5

[34] Lee, J., and Santavicca, D., "Experimental Diagnostics for the Study of Combustion Instabilities in Lean Premixed Combustors," Journal of Propulsion and Power, Vol. 19, 2003, pp. 735-750. doi:10.2514/2.6191

[35] Ayoola, B., Balachandran, R., Frank, J., Mastorakos, E., and Kaminski, C., "Spatially Resolved Heat Release Rate Measurements in Turbulent Premixed Flames," Combustion and Flame, Vol. 144, 2006, pp. 1-16. doi:10.1016/j.combustflame.2005.06.005

[36] Lauer, M., and Sattelmayer, T., "On the Adequacy of Chemiluminescence as a Measure for Heat Release in Turbulent Flames with Mixture Gradients," Journal of Engineering for Gas Turbines and Power, Vol. 132, 2010, Paper 061502. doi:10.1115/1.4000126

[37] Jolliffe, I. T., Principal Component Analysis, Springer, New York, 2002.

[38] Berkooz, G., Holmes, P., and Lumley, J. L., "The Proper Orthogonal Decomposition in the Analysis of Turbulent Flows," Annual Review of Fluid Mechanics, Vol. 25, No. 1, 1993, pp. 539-575. doi:10.1146/annurev.fl.25.010193.002543

[39] Huang, Y., Wang, S., and Yang, V., "A Systematic Analysis of Combustion Dynamics in a Lean Premixed Swirl-Stabilized Combustor," AIAA Journal, Vol. 44, 2006, pp. 724-740. doi: $10.2514 / 1.15382$

[40] Danby, S. J., and Echekki, T., "Proper Orthogonal Decomposition Analysis of Autoignition Simulation DATA OF Nonhomogeneous 
Hydrogen/Air Mixtures," Combustion and Flame, Vol. 144, 2006, pp. 126-138.

doi:10.1016/j.combustflame.2005.06.014

[41] Duwig, C., and Fuchs, L., "Large Eddy Simulation of Vortex Breakdown/Flame Interaction," Physics of Fluids, Vol. 19, 2007, Paper 075103. doi:10.1063/1.2749812

[42] Chakraborty, N., and Hawkes, E. R., "Determination of 3-D Flame Surface Density Variables from 2D Measurements: Validation Using Direct Numerical Simulation," Physics of Fluids, Vol. 23, 2011, Paper 065113.

doi:10.1063/1.3601483

[43] Jeong, J., and Hussain, F., "On the Identification of a Vortex," Journal of Fluid Mechanics, Vol. 285, 1995, pp. 69-94. doi: $10.1017 / \mathrm{S} 0022112095000462$
[44] Chong, M. S., Perry, A. E., and Cantwell, B. J., "A General Classification of Three-Dimensional Flow Fields," Physics of Fluids A, Vol. 2, 1990, pp. 765-777. doi: $10.1063 / 1.857730$

[45] Zhou, J., Adrian, R. J., Balachandar, S., and Kendall, T. M., "Mechanisms for Generating Coherent Packets of Hairpin Vortices," Journal of Fluid Mechanics, Vol. 387, 1999, pp. 353-396. doi:10.1017/S002211209900467X

[46] Chakraborty, P., Balachandar, S., and Adrian, R. J., "On the Relationships Between Local Vortex Identification Schemes," Journal of Fluid Mechanics, Vol. 535, 2005, pp. 189-214. doi:10.1017/S0022112005004726

E. Gutmark Associate Editor 